\title{
Dual-plane stereo particle image velocimetry measurements of velocity gradient tensor fields in turbulent shear flow. II. Experimental results
}

\author{
John A. Mullin and Werner J. A. Dahm ${ }^{\text {a) }}$ \\ Laboratory for Turbulence \& Combustion (LTC), Department of Aerospace Engineering, \\ The University of Michigan, Ann Arbor, Michigan 48109-2140
}

(Received 22 July 2004; accepted 26 September 2005; published online 9 March 2006)

\begin{abstract}
Results are presented from highly resolved dual-plane stereo particle image velocimetry (DSPIV) measurements for the structure, statistics, similarity, and scaling of all nine simultaneous components of the velocity gradient tensor fields $\partial u_{i} / \partial x_{j}$ on the quasi-universal intermediate and small scales of turbulent shear flows. Measurements were obtained at three combinations of the outer-scale Reynolds number $\operatorname{Re}_{\delta}$ and the local mean shear rate $S$ in the fully developed self-similar far field of a turbulent jet, and thus reflect the combined effects of the large-scale structure, spatial inhomogeneities, and anisotropies inherent in such a flow. Conditions addressed in this study correspond to local outer-scale Reynolds numbers $\operatorname{Re}_{\delta}=6,000$ and 30,000 and local mean shear values $S \delta / u_{c}=0$ and 1.7, corresponding to Taylor-scale Reynolds numbers $\operatorname{Re}_{\lambda} \approx 44$ and 113 and shear rates $S k / \varepsilon=0$ and 2.1. Gradient fields investigated here include the individual velocity gradient component fields, the strain rate component fields and the associated principal strain rates, the vorticity component fields and their orientations with respect to the principal strain axes, the enstrophy and enstrophy production rate fields, and the true kinetic energy dissipation rate field. Results normalized on both inner- and outer-scale variables are presented to allow interpretation relative to the similarity and scaling implied by classical turbulence theory. For both $\operatorname{Re}_{\delta}$ values at $S=0$, results show that most aspects of these gradient fields are essentially in agreement with the predictions from homogeneous isotropic turbulence, while for $S \neq 0$ there are significant and consistent departures from isotropy. Results also provide direct measurements of the exponential scaling factors in the left and right tails of the velocity gradient distributions, as well as quantification of the inner (viscous) length scales in the enstrophy and dissipation rate fields. In addition, strong evidence for multifractal scale similarity at length scales greater than about twice the viscous length $\lambda_{\nu}$ is found in both the enstrophy and dissipation rate fields. () 2006 American Institute of Physics. [DOI: 10.1063/1.2166448]
\end{abstract}

\section{INTRODUCTION}

Turbulence theory has been extensively developed for homogeneous isotropic and uniformly sheared turbulence. Its application to inhomogeneous turbulent shear flows is based, in broadest terms, on the classical hypothesis of a universal approach to the homogeneous and isotropic state at sufficiently small scales. Beyond this local isotropy assumption, however, the precise extension of classical theory to the turbulence encountered in shear flows is still a subject of considerable uncertainty. Any universal approach to the homogeneous, isotropic state at small scales in turbulent shear flows is complicated by the presence of organized large-scale vortical structures, spatial inhomogeneities, anisotropy, and the comparatively small range of scales present at the moderate Reynolds numbers in many experimental studies. Relatively little is known about the range of scales over which these characteristics of shear flow turbulence will create significant departures from the asymptotic state that is presumed to apply at sufficiently small scales. Similarly, comparatively little is known about the effect of the Reynolds

\footnotetext{
a) Author to whom correspondence should be addressed. Electronic mail: wdahm@umich.edu
}

number and the local mean shear rate on this asymptotic approach, or about the departures from the universal state at larger scales. Several studies have reported comparatively large degrees of anisotropy at remarkably small scales in shear flow turbulence. ${ }^{1-4}$ Here we present results from an experimental study that characterizes key aspects of the structure, statistics, similarity and scaling of velocity gradient fields at the intermediate and small scales of a turbulent free shear flow for several values of the local Reynolds number $\operatorname{Re}_{\delta}$ and the local mean shear rate $(S k / \varepsilon)$. We use these to infer the relevance of the local isotropy hypothesis to the small scales of turbulent shear flows at relatively moderate $\operatorname{Re}_{\delta}$ values, and to quantify certain aspects of the departures from this isotropic state at these values of $\operatorname{Re}_{\delta}$ and $(S k / \varepsilon)$.

Classical theory is usually applied to turbulent shear flows by scaling turbulence quantities with the local rms velocity fluctuation $u_{\mathrm{rms}}^{\prime}$ and local Taylor scale $\lambda_{g}$, or equivalently by corresponding scalings with local values of the average turbulence kinetic energy $k$ and average turbulent dissipation rate $\varepsilon$. However these parameters are themselves characteristics of the turbulence, and thus can be related to appropriate outer length and velocity scales associated with the mean flow. Since kinetic energy is supplied from the local mean flow to the turbulence through vortex line stretch- 
ing by the mean shear profile, and since the local mean flow width typically increases only slowly with downstream distance $x$, appropriate outer-scale parameters are the local velocity $u_{c}(x)$ and length $\delta(x)$ that characterize the local mean shear profile. In jets and plumes, for example, $u_{c}(x)$ and $\delta(x)$ are the local mean centerline velocity and the local flow width, so that the peak mean shear is $O\left(u_{c} / \delta\right)$, while in mixing layers the outer velocity and length scales are the freestream velocity difference and the local flow width. Scaling laws for $u_{c}$ and $\delta$ can often be obtained by simple dimensional arguments, allowing local turbulence properties to be obtained from their respective scalings in these outer-scale parameters if the corresponding scaling coefficients are known. One of the goals of the present study has been to determine these outer-variable scaling coefficients for various key turbulence quantities in a turbulent shear flow.

Aspects of turbulent free shear flows associated with the outer scales, such as mean velocities and gradients of mean quantities, scale with the outer velocity and length scales $u_{c}(x)$ and $\delta(x)$. The corresponding local outer-scale Reynolds number $\operatorname{Re}_{\delta}(x) \equiv u_{c} \delta / \nu$ then determines the local inner (viscous) length scale $\lambda_{\nu}(x)$ via the inner-to-outer length scale relation $\left(\lambda_{v} / \delta\right) \sim \mathrm{Re}_{\delta}^{-3 / 4}$. Quantities that are concentrated at the small scales of the flow, such as velocity gradients, scale with the inner length scale $\lambda_{\nu}$ and the inner velocity scale $\left(\nu / \lambda_{\nu}\right)$; e.g., the rms fluctuation in the enstrophy would scale as $\nu / \lambda_{\nu}^{2}$. When the resulting inner-variable scalings are expressed in terms of the outer-scale parameters $u_{c}$ and $\delta$, they imply specific $\operatorname{Re}_{\delta}$ scalings, e.g., the enstrophy scaling above becomes $\left(u_{c} / \delta\right) \operatorname{Re}_{\delta}^{1 / 2}$. One of the goals of the present study has been to determine the extent to which the outer- and inner-variable scalings predicted by classical theory apply to velocity gradient quantities at these $\operatorname{Re}_{\delta}$ values in turbulent free shear flows, including strain rates and vorticity components, the enstrophy and its production rate, and the kinetic energy dissipation rate. This study has also sought to directly determine the corresponding scaling coefficients in the outerand inner-variable scalings of these quantities, and to characterize the quasi-universal distributions of the resulting scaled velocity gradient quantities in turbulent shear flows.

The competing effects of the local strain rates (which act to reduce the gradient length scales) and viscous diffusion (which acts to increase the gradient scales) reach an equilibrium at the strain-limited viscous length scale $\lambda_{\nu}$ in the velocity gradient fields. A corresponding strain-diffusion competition involving the diffusivity $D$ of conserved scalars leads to the strain-limited scalar diffusion length scale $\lambda_{D}$ $=\lambda_{\nu} \mathrm{Sc}^{-1 / 2}$, where $\mathrm{Sc} \equiv \nu / D$, is the scalar gradient field. ${ }^{5,6} \mathrm{By}$ expressing the local inner-to-outer length scale relation $\lambda_{\nu} / \delta=\Lambda \mathrm{Re}_{\delta}^{-3 / 4}$ in terms of the local outer-scale Reynolds number $\operatorname{Re}_{\delta}$, the scaling coefficient $\Lambda$ should presumably be the same for all turbulent free shear flows. Indirect measurements of $\Lambda$ based on the gradient length scales $\lambda_{D}$ in scalar gradient fields $^{6-9}$ have indicated $\langle\Lambda\rangle \approx 11.2$. Here $\lambda_{D}$ is the physical thickness of the scalar diffusion layers, defined from the layer-normal scalar dissipation profile as the distance between the points where the dissipation drops to $20 \%$ of the local layer-normal maximum value..$^{5-9}$ Note that $\lambda_{\nu}$ is proportional to the Kolmogorov length scale $\lambda_{K} \equiv\left(\nu^{3} \varepsilon\right)^{1 / 4}$.
However $\lambda_{K}$ is defined solely on dimensional grounds, and thus gives the correct scaling for the viscous diffusion length scale but does not give the physical scale $\lambda_{\nu}$ at which the competing effects of strain and viscous diffusion balance to set the local strain-limited viscous diffusion length scale. Surrogate dissipation measurements ${ }^{10}$ and $\langle\Lambda\rangle$ as above suggest $\lambda_{\nu} \simeq 5.9 \lambda_{K}$, which appears at least roughly consistent with the peak in the dissipation spectrum. Among the goals of this investigation has been to determine the distribution of $\Lambda$ values directly from velocity gradient measurements, and to determine the factor relating $\lambda_{\nu}$ and $\lambda_{K}$ directly from the measured kinetic energy dissipation rate fields.

The classical assumption of local isotropy, when applied to turbulent shear flows, suggests that if the local outer-scale Reynolds number $\operatorname{Re}_{\delta}$ is large enough for a sufficient scale separation to exist between the local inner and outer length scales $\lambda_{\nu}$ and $\delta$, then at sufficiently small scales the structure and statistics of all turbulent shear flows at all values of $\operatorname{Re}_{\delta}$ should be identical when scaled on the inner variables $\nu$ and $\lambda_{\nu}$. Moreover, with increasingly smaller scales the structure, statistics, similarity and scaling of all velocity gradients should approach the corresponding characteristics of homogeneous isotropic turbulence. One of the further goals of the present study has been to determine the extent to which the outer- and inner-scale similarities and scalings predicted by classical theory apply to various velocity gradient fields at these $\operatorname{Re}_{\delta}$ values in turbulent shear flows.

Refinements to these implications of classical theory focus on relatively weak additional $\operatorname{Re}_{\delta}$ scalings associated with intermittency effects. The resulting intermittency corrections involve various assumptions for the physical structure and scaling of velocity gradient fields at intermediate and small scales of high Reynolds number turbulent flows. ${ }^{2,11,12}$ However most studies to date of such intermittency effects in turbulent shear flows have been based on indirect measurements of velocity and scalar gradient quantities. In most cases, these apply Taylor's hypothesis to single-point time-series data $u(t)$ to obtain a surrogate estimate of the form $(\partial u / \partial t)^{2}$ that approximates one-dimensional intersections through the dissipation rate fields. ${ }^{13}$ One of the further goals of this study has been to characterize intermittency effects from the present direct measurements of true velocity gradient fields in turbulent shear flows, avoiding any resort to Taylor's hypothesis or to surrogate enstrophy or dissipation fields.

The present investigation is based on highly resolved direct experimental measurements of the structure, statistics, similarity, and scaling of all nine simultaneous components of the velocity gradient tensor fields $\partial u_{i} / \partial x_{j}$ on the quasiuniversal intermediate and small scales of turbulent shear flows. Unlike direct numerical simulations (DNS) of homogeneous isotropic or uniformly sheared turbulence in periodic domains, the present results from a turbulent shear flow reflect the combined effects of the large-scale structure, inhomogeneities, and anisotropies inherent in such a flow, and address Reynolds numbers that significantly exceed values currently attainable by DNS of spatially developing turbulent shear flows. Three combinations of the local outer-scale Reynolds number $\operatorname{Re}_{\delta}$ and the local mean shear rate $S$ 
$\equiv\left(2 S_{i j} S_{i j}\right)^{1 / 2}$ are investigated here to examine their effect on the similarity and scaling implied by classical turbulence theory for the intermediate and small scales of turbulent shear flows. Results are presented for $\operatorname{Re}_{\delta}=6,000$ and 30,000 and for values of the outer-scaled mean shear rate $\left(S \delta / u_{c}\right)$ $=0$ and 1.7. These correspond to Taylor-scale Reynolds numbers $\operatorname{Re}_{\lambda}=44$ and 113, and to normalized mean shear rates $(S k / \varepsilon)=0$ and 2.1. The two mean shear rates are, respectively, from measurements on the centerline of an axisymmetric turbulent jet and at the radial location of maximum shear in the same flow. The latter value of $(S k / \varepsilon)$ is typical of the peak mean shear rate in most free turbulent shear flows. It is also comparable to the $(S k / \varepsilon) \approx 3.2$ value that applies throughout the log layer of wall-bounded turbulent shear flows. Direct numerical simulations of homogeneous uniformly sheared turbulence ${ }^{14}$ have examined roughly comparable shear rates, corresponding to $(S k / \varepsilon) \leqslant 4.3$. Experimental studies of homogeneous uniformly sheared turbulence have addressed $(S k / \varepsilon)$ values ranging from 6.5 (Ref. 15), 13 (Ref. 3), and 34 (Ref. 16), however such comparatively larger shear values are principally relevant to the viscous near-wall region $(y+<20)$ of wall-bounded shear flows.

The present study investigates the effects of these parameters on various aspects of the velocity gradient component fields, the strain rate component fields and their associated eigenvalues and eigenvectors, the vorticity component fields and their orientations with respect to the principal strain axes, the enstrophy and enstrophy production rate fields, and the true kinetic energy dissipation rate field. The results focus principally on (i) assessments of the $\mathrm{Re}_{\delta}$ similarity and scaling in various probability densities associated with these fields for the $S=0$ and $S \neq 0$ cases; (ii) determination of exponential scaling factors in the tails of these distributions; (iii) determination of the quasi-universal scaling coefficients in inner- and outer-variable scaling laws associated with these fields; (iv) comparisons with previous experimental and computational results for velocity gradients in homogeneous, isotropic turbulence at comparable $\mathrm{Re}_{\lambda}$ values; (v) measurements of the inner length scales in the enstrophy and dissipation rate fields; and (vi) assessments of scale similarity in the true enstrophy and kinetic energy dissipation rate fields. Results are presented with both local inner- and outerscale normalizations to allow their interpretation relative to classical theory and their application to turbulent free shear flows at other values of $\operatorname{Re}_{\delta}$ and $\left(S \delta / u_{c}\right)$.

\section{EXPERIMENTAL ARRANGEMENT}

\section{A. Dual-plane stereo particle imaging velocimetry (DSPIV)}

Two-frequency dual-plane stereo particle image velocimetry ${ }^{17,18}$ was here used to obtain highly resolved direct noninvasive measurements of all nine components of the velocity gradient tensor field $\partial u_{i} / \partial x_{j}$ at the intermediate and small scales of a turbulent shear flow. The approach is based on measurements in two differentially spaced light-sheet planes, as shown in Figs. 1 and 2 of Ref. 18, using two different laser frequencies in conjunction with filters to sepa-
TABLE I. Parameters used in inner- and outer-variable normalizations in the present study.

\begin{tabular}{cccc}
\hline \hline & $\operatorname{Re}_{\delta}=6,000$ & $\operatorname{Re}_{\delta}=6,000$ & $\operatorname{Re}_{\delta}=30,000$ \\
\cline { 2 - 4 } Parameter & $\operatorname{Re}_{\lambda}=45$ & $\operatorname{Re}_{\lambda}=43$ & $\operatorname{Re}_{\lambda}=113$ \\
$N$ & $S=0$ & $S=1.2$ & $S=0$ \\
\hline$u_{c}(\mathrm{~m} / \mathrm{s})$ & 1244 & 150 & 1019 \\
$\delta(\mathrm{m})$ & 1.2 & 1.2 & 3.7 \\
$\lambda_{\nu}(\mathrm{mm})$ & 0.075 & 0.075 & 0.129 \\
$\lambda_{g}(\mathrm{~mm})$ & 1.24 & 1.70 & 0.64 \\
$u_{\mathrm{rms}}^{\prime}(\mathrm{m} / \mathrm{s})$ & 2.48 & 3.79 & 1.91 \\
$v_{\mathrm{rms}}^{\prime}(\mathrm{m} / \mathrm{s})$ & 0.24 & 0.16 & 0.83 \\
$w_{\mathrm{rms}}^{\prime}(\mathrm{m} / \mathrm{s})$ & 0.20 & 0.11 & 0.64 \\
$\varepsilon\left(\mathrm{m}^{2} / \mathrm{s}^{3}\right)$ & 0.21 & 0.13 & 0.69 \\
$\nu\left(\mathrm{m}^{2} / \mathrm{s}\right)$ & 1.76 & 0.47 & 27.7 \\
\hline \hline
\end{tabular}

rate the light scattered from the seed particles onto the individual stereo camera pairs. The differential spacing of the two stereo PIV planes allows resolution of velocity gradient fields to the local inner scale $\lambda_{\nu}$ of the turbulent flow.

Briefly, the present arrangement consisted of four $\mathrm{Nd}$ :YAG lasers, of which two were sequentially triggered to create the double pulses for the $532 \mathrm{~nm}$ light sheets and the other two, also sequentially triggered at the same instants of time, pumped two pulsed dye lasers that provided the 635 $\mathrm{nm}$ light sheets. A knife edge was traversed across each sheet and the transmitted light collected onto a photodiode detector to quantitatively establish the $400 \pm 20 \mu \mathrm{m}$ thicknesses of the two $532 \mathrm{~nm}$ light sheets and the $800 \pm 20 \mu \mathrm{m}$ thicknesses of the two $635 \mathrm{~nm}$ light sheets (see Fig. 4 of Ref. 17). An optical technique that images each sheet cross section and determines the sheet centerline based on the measured intensity profiles at various elevations across the sheet and over the field of view was used to quantitatively verify coincidence and parallelism, to within a rms angular error of less than $0.2^{\circ}$, of the two $532 \mathrm{~nm}$ and $635 \mathrm{~nm}$ light-sheet pairs, and to confirm the $400 \pm 20 \mu \mathrm{m}$ separation between the sheets (see Fig. 5 of Ref. 17).

The scattered light from $0.5 \mu \mathrm{m}$ aluminum oxide seed particles was recorded on four 12-bit $1280 \times 1024$-pixel CCD cameras arranged using Sigma 70-300 $f$ 4-5.6 APO macro lenses to achieve the desired $15.5 \mathrm{~mm} \times 12.5 \mathrm{~mm}$ field of view. The $532 \mathrm{~nm}$ camera pair had narrow-band filters centered at $532 \pm 5 \mathrm{~nm}$ that blocked the $635 \mathrm{~nm}$ light, and the $635 \mathrm{~nm}$ cameras were equipped with OG570 Schott glass filters that blocked the $532 \mathrm{~nm}$ light. The four cameras were arranged in an asymmetric, forward-forward scatter, angular-displacement configuration with each of the camera pairs satisfying the Scheimpflug condition for stereoscopic imaging. Each pair of stereo cameras was orientated with an included angle of $50^{\circ}$ between the optical axes of each camera pair, as shown in Fig. 6 of Ref. 17.

Particle images were processed with an adaptive multipass technique using $32 \times 32$-pixel interrogation boxes with no overlap in the final vector fields, giving the same in-plane resolution between vectors as the out-of-plane resolution be- 

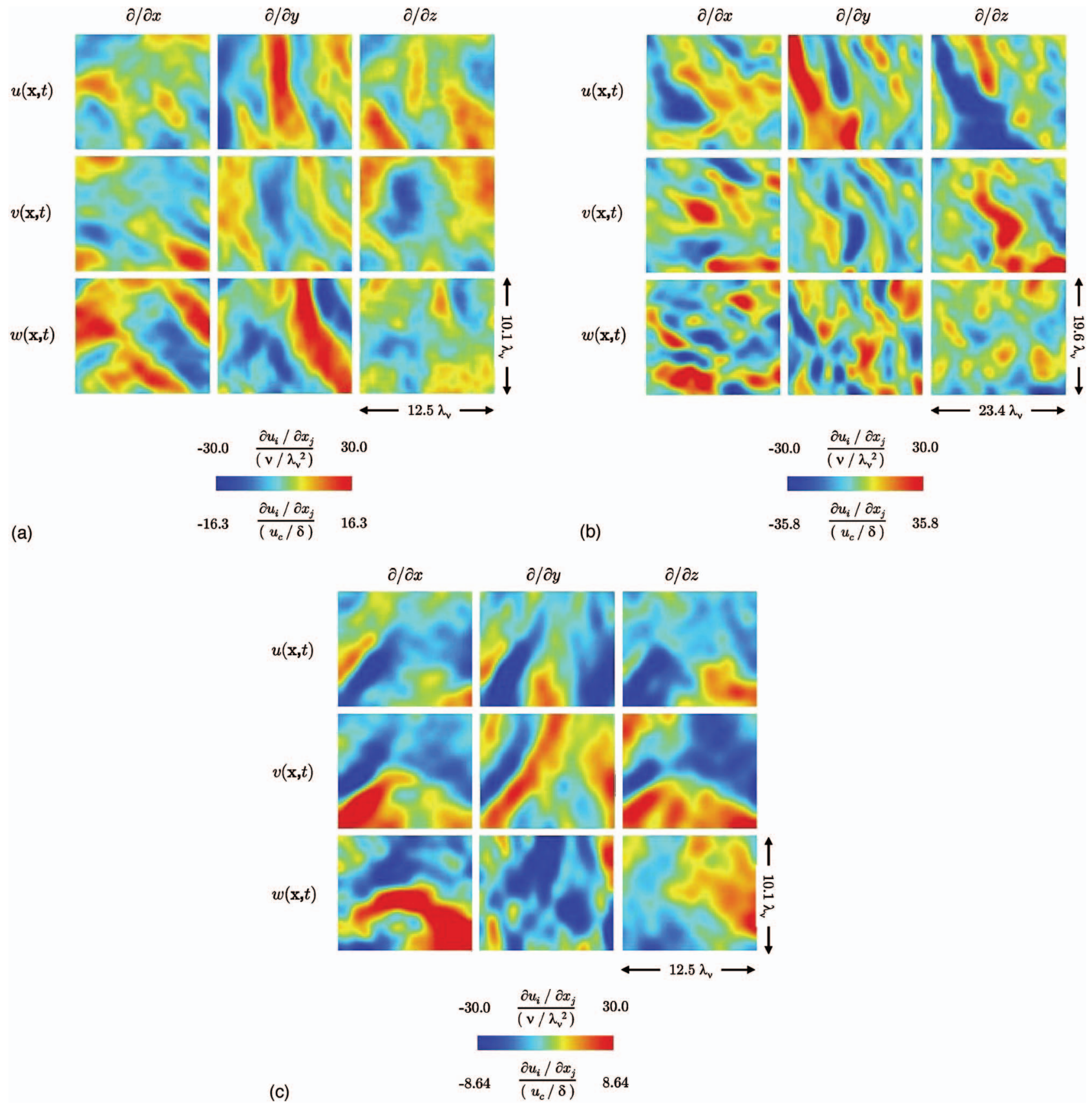

FIG. 1. (Color) Typical nine-component velocity gradient tensor fields $\partial u_{i} / \partial x_{j}(\mathbf{x}, t)$ at $\left(\operatorname{Re}_{\delta} ; S\right)=(\mathrm{a})(6,000 ; 0)$, (b) $(30,000 ; 0)$, and (c) $(6,000 ; 1.2)$. Field of view is shown normalized by local inner length scale $\lambda_{\nu}$. Color scale shows quantitative values normalized both on local inner $\left(\nu, \lambda_{\nu}\right)$ and outer $\left(u_{c}, \delta\right)$ scales.

tween the planes. Velocity gradients were computed using linear central differencing in the $x$ and $y$ directions and onesided differencing in the $z$ direction of the measured velocity fields to obtain all nine components of $\partial u_{i} / \partial x_{j}$. The overall spatial resolution in the resulting velocity gradient fields was determined by the laser sheet thicknesses and separation and the PIV processing to be $1100 \mu \mathrm{m}$. Extensive assessments of the accuracy of velocity gradient fields obtained with this DSPIV technique are given in Ref. 18, where the resulting measured divergence values demonstrate overall rms errors of $8.9 \%$ and $11.7 \%$ in the off- and on-diagonal components of the measured velocity gradients $\partial u_{i} / \partial x_{j}$.

\section{B. Flow configuration}

The present DSPIV measurements were obtained on the centerline and at the radial location of maximum mean shear in the self-similar far field of an axisymmetric coflowing turbulent jet. Air seeded with $0.5 \mu \mathrm{m}$ aluminum oxide particles issued from a $1.0 \mathrm{~m}$ long tube with $2.2 \mathrm{~mm}$ inner diameter at nominal exit velocity $U_{o}=12.7 \mathrm{~m} / \mathrm{s}$ for the $\operatorname{Re}_{\delta}$ $=6,000$ cases, and $U_{o}=36.7 \mathrm{~m} / \mathrm{s}$ for the $\operatorname{Re}_{\delta}=30,000$ case, into a uniformly seeded coflowing air stream in a 30 $\times 30 \mathrm{~cm}$ test section with coflow velocity $U_{\infty}=0.25 \mathrm{~m} / \mathrm{s}$. The measurements for the $\operatorname{Re}_{\delta}=6,000$ cases were obtained 
$17 \mathrm{~cm}$ downstream from the virtual origin of the jet, and 29 $\mathrm{cm}$ downstream for the $\operatorname{Re}_{\delta}=30,000$ case. In terms of the invariant momentum radius $\vartheta$ that governs the similarity scaling of coflowing turbulent jets, ${ }^{19,20}$ these locations respectively correspond to $(x / \vartheta)=2.1$ and 1.0 , both of which are within the jet-limit scaling of coflowing jets in which the effect of the coflow is negligible. Conventional PIV measurements of the resulting mean velocity and velocity fluctuation profiles, given in Figs. 3 and 4 of Ref. 18, show good agreement with previously reported results for jet-limit scaling and served to validate the turbulent shear flow used in these experiments. These also provided the mean centerline excess velocity $u_{c}(x)=U_{c}(x)-U_{\infty}$ and the local flow width $\delta(x)$ for each case as given in Table I. Further details of the flow facility are given in Ref. 21.

For the $\left(S \delta / u_{c}\right)=1.7$ case, the DSPIV imaging region was centered at the point of maximum shear in the local mean velocity profile. For the two $\left(S \delta / u_{c}\right)=0$ cases this was centered on the flow centerline, where the mean shear is zero, but extended $7.25 \mathrm{~mm}$ on either side of the centerline, with the result that effects of the relatively small mean shear $\left(S \delta / u_{c}<0.2\right)$ present on either side of the centerline will be detectable in these results.

Although these measurements were obtained in the selfsimilar far field of an axisymmetric coflowing turbulent jet, the $15.5 \mathrm{~mm} \times 12.5 \mathrm{~mm}$ field of view of the measurements was significantly smaller than the local outer scale $\delta(x)$ of the flow, and was of the order of 10-20 local inner length scales $\lambda_{\nu}(x)$ in each direction. As a result, if the local outerscale Reynolds numbers $\operatorname{Re}_{\delta}$ are large enough for a sufficient scale separation to exist between the local outer and inner length scales, then when scaled on inner variables the results should be independent of both the particular Reynolds numbers and the particular shear flow in which they were obtained, and should be representative of the generic structure, statistics, similarity and scaling of velocity gradient fields at the intermediate and small scales of all high Reynolds number turbulent free shear flows at these values of the mean shear rate $\left(S \delta / u_{c}\right)$.

\section{VELOCITY GRADIENT FIELDS}

Figures 1(a)-1(c) show examples of the simultaneously measured instantaneous velocity gradient tensor component fields $\partial u_{i} / \partial x_{j}$ at the three combinations of $\operatorname{Re}_{\delta}$ and $\left(S \delta / u_{c}\right)$ considered in this study. The dimensions of each plane are indicated in terms of the local inner length scale $\lambda_{\nu}$, namely the local strain-limited viscous diffusion length scale obtained via $\lambda_{\nu} / \delta=\Lambda \operatorname{Re}_{\delta}^{-3 / 4}$ with $\Lambda \approx 11.2$ as noted in Sec. I. The two measurement planes are separated in the $z$ direction by $400 \mu \mathrm{m}$, with the resulting $\left(\Delta z / \lambda_{\nu}\right)=0.32$ in the two $\operatorname{Re}_{\delta}=6,000$ cases and $\left(\Delta z / \lambda_{\nu}\right)=0.63$ in the $\operatorname{Re}_{\delta}=30,000$ case. The velocity gradient fields were obtained by central differencing of the velocity component values within each $x-y$ plane and one-sided differencing between the two $z$ planes. The differencing template was based on the pixel-based coordinate frame as well as the two additional frames resulting from $45^{\circ}$ rotations around the $x$ and $z$ axes. This makes use of all 18 points in the $3 \times 3 \times 2$ volume around each point to
TABLE II. Moment values scaled on inner variables $\left(\nu, \lambda_{\nu}\right)$ for all nine components of the velocity gradient field $\partial u_{i} / \partial x_{j}$ measured at $\left(\operatorname{Re}_{\delta} ; S\right)$ $=(6,000 ; 0)$, for which $\operatorname{Re}_{\lambda}=45$, showing mean $M_{1}$, rms fluctuation $M_{2}$, skewness $M_{3}$, and kurtosis $M_{4}$.

\begin{tabular}{ccccc}
\hline \hline $\begin{array}{c}\text { Derivative } \\
\text { component }\end{array}$ & $M_{1}$ & $M_{2}$ & $M_{3}$ & $M_{4}$ \\
\hline$\partial u / \partial x$ & -0.018 & 7.153 & -0.428 & 4.222 \\
$\partial u / \partial y$ & 0.002 & 9.944 & -0.007 & 4.635 \\
$\partial u / \partial z$ & 0.008 & 9.639 & -0.220 & 6.021 \\
$\partial v / \partial x$ & -0.004 & 9.233 & 0.022 & 4.834 \\
$\partial v / \partial y$ & -0.022 & 6.707 & -0.384 & 3.817 \\
$\partial v / \partial z$ & 0.009 & 9.637 & -0.168 & 4.858 \\
$\partial w / \partial x$ & -0.013 & 9.850 & 0.031 & 4.483 \\
$\partial w / \partial y$ & -0.017 & 9.539 & -0.027 & 4.656 \\
$\partial w / \partial z$ & -0.018 & 6.929 & -0.137 & 4.526 \\
\hline \hline
\end{tabular}

give more accurate derivatives without degrading the spatial resolution in the resulting gradient fields. As shown in Table I, over 1000 such validated velocity gradient planes were measured in each of the $\operatorname{Re}_{\delta}=6,000$ and 30,000 cases with $\left(S \delta / u_{c}\right)=0$.

The color scales in Figs. 1(a)-1(c) give the resulting velocity gradient component values normalized by the local outer-scale value $\left(u_{c} / \delta\right)$ and by the local inner-scale value $\left(\nu / \lambda_{\nu}^{2}\right)$. From $\lambda_{\nu} / \delta \sim \operatorname{Re}_{\delta}^{-3 / 4}$ the inner-scaled values should then be $\operatorname{Re}_{\delta}$ independent, while the outer-scaled values will increase as $\operatorname{Re}_{\delta}^{1 / 2}$. Comparing the color scales for the two $S$ $=0$ cases at $\operatorname{Re}_{\delta}=6,000$ and 30,000 in Figs. 1 (a) and $1(\mathrm{~b})$ shows that this is very nearly the case. The range of innerscaled values is the same despite the different $\operatorname{Re}_{\delta}$ values, while the range of outer-scaled values increases with $\mathrm{Re}_{\delta}$, reflecting the increasing concentration of velocity gradient fields onto an increasingly smaller fraction of the volume as $\operatorname{Re}_{\delta}$ is increased.

\section{A. Taylor scales and $\mathrm{Re}_{\lambda}$ values}

Taylor-scale Reynolds numbers allow comparisons of the present results with corresponding investigations of homogeneous isotropic or uniformly sheared turbulence. The Taylor scale can be obtained several ways, all of which are equivalent in homogeneous isotropic turbulence but which give slightly different results in turbulent shear flows. Parabolic fits to the two-point longitudinal and transverse velocity fluctuation correlations, $f(r)$ and $g(r)$, from the measured velocities in the most highly resolved case, with $\operatorname{Re}_{\delta}$ $=6,000$ and $\left(S \delta / u_{c}\right)=0$, give corresponding Taylor scales $\lambda_{f} \approx 4.5 \mathrm{~mm}$ and $\lambda_{g} \approx 3.3 \mathrm{~mm}$. The ratio of these is roughly consistent with the $\sqrt{2}$ factor applicable in homogeneous isotropic turbulence. Alternatively, isotropy relations of the form $\lambda_{g}^{2}=\overline{u^{\prime 2}} / \overline{(\partial u / \partial x)^{2}}$, together with the measured $M_{2}$ values in Table II, give $\lambda_{f} \approx 4.4 \mathrm{~mm}$ and $\lambda_{g} \approx 3.2 \mathrm{~mm}$. However, since the kinetic energy dissipation rate fields $2 \nu S_{i j} S_{i j}(\mathbf{x}, t)$ in Sec. VI were measured directly without any assumption of isotropy, Taylor scales can also be obtained from the resulting mean dissipation rates $\varepsilon$ via the relation $\lambda_{g}^{2}=15 \overline{u^{\prime 2}}(\nu / \varepsilon)$. The two $\operatorname{Re}_{\delta}=6,000$ cases, with $\left(S \delta / u_{c}\right)=0$ and 1.7, are essentially fully resolved and thus their mea- 

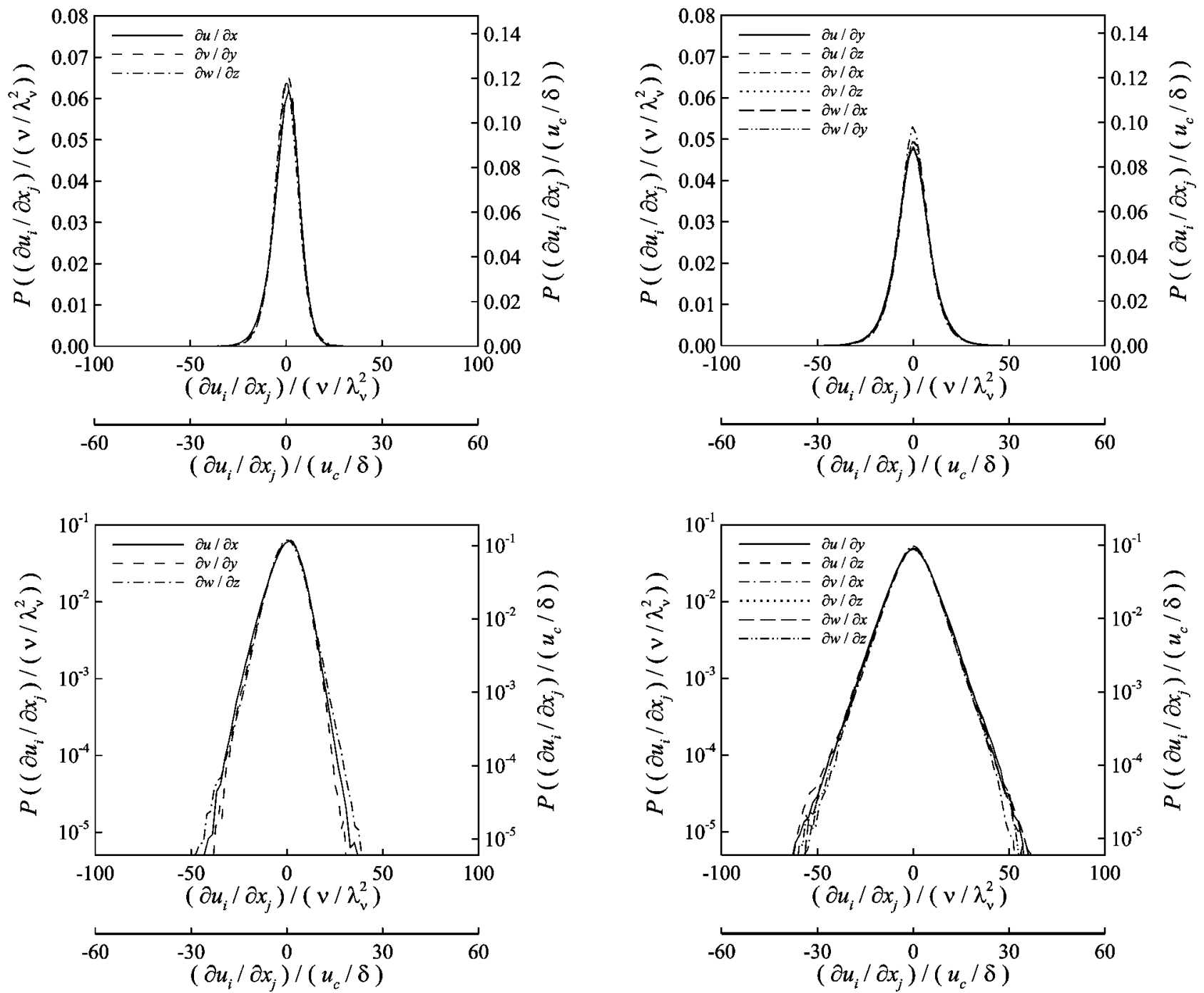

FIG. 2. Probability densities for (left) on-diagonal $(i=j)$ and (right) off-diagonal $(i \neq j)$ components of $\partial u_{i} / \partial x_{j}$ at $\left(\operatorname{Re}_{\delta} ; S\right)=(6,000 ; 0)$ in linear $($ top $)$ and semilogarithmic (bottom) axes scaled on local inner $\left(\nu, \lambda_{\nu}\right)$ and outer $\left(u_{c}, \delta\right)$ variables. Resulting similarity suggests near-isotropy in velocity gradient fields.

sured mean dissipation rates $\varepsilon=1.757 \mathrm{~m}^{2} / \mathrm{s}^{3}$ and $0.465 \mathrm{~m}^{2} / \mathrm{s}^{3}$ in Table I together with their respective $\mathrm{rms}$ velocity fluctuation values can be used to determine $\lambda_{g}$ values. Doing this gives $\lambda_{g}=2.48 \mathrm{~mm}$ for the $\left(S \delta / u_{c}\right)=0$ case, and $\lambda_{g}=3.79 \mathrm{~mm}$ for the $\left(S \delta / u_{c}\right)=1.7$ case.

For the $\operatorname{Re}_{\delta}=30,000$ case with $\left(S \delta / u_{c}\right)=0$, the slightly lower relative spatial resolution in the velocity gradient measurements suggests that the resulting value for the mean dissipation rate $\varepsilon \approx 27.67 \mathrm{~m}^{2} / \mathrm{s}^{3}$ is slightly low. If this is nevertheless used, it gives $\lambda_{g} \approx 2.07 \mathrm{~mm}$. However the Taylor scale for this case can also be obtained from the $\operatorname{Re}_{\delta}$ $=6,000$ case with $\left(S \delta / u_{c}\right)=0$ by the scaling relation $\lambda_{g} / \delta$ $\sim \operatorname{Re}_{\delta}^{-1 / 2}$. From the $\delta$ values in Table $\mathrm{I}$ and $\lambda_{g}$ above this gives $\lambda_{g}=1.91 \mathrm{~mm}$ at $\operatorname{Re}_{\delta}=30,000$. This is only slightly smaller than the $\lambda_{g}$ obtained above, consistent with the fact that the $\operatorname{Re}_{\delta}=30,000$ case is only slightly less than fully resolved. Since the $\operatorname{Re}_{\delta}=6,000$ case is essentially fully resolved, the Taylor scale at $\operatorname{Re}_{\delta}=30,000$ obtained from it via this scaling approach should be the more accurate value.

From these $\lambda_{g}$ values and the measured rms velocity fluctuations in Table I, corresponding Taylor-scale Reynolds numbers $\operatorname{Re}_{\lambda} \equiv u_{\mathrm{rms}}^{\prime} \lambda_{g} / \nu$ are $\operatorname{Re}_{\lambda} \approx 45$ for the $\operatorname{Re}_{\delta}=6,000$ case with $\left(S \delta / u_{c}\right)=0, \operatorname{Re}_{\lambda} \approx 43$ for the $\operatorname{Re}_{\delta}=6,000$ case with $\left(S \delta / u_{c}\right)=1.7$, and $\operatorname{Re}_{\lambda} \approx 113$ for the $\operatorname{Re}_{\delta}=30,000$ case with $\left(S \delta / u_{c}\right)=0$. Note that the two $\operatorname{Re}_{\delta}=6,000$ cases are at $\operatorname{Re}_{\lambda}$ values comparable to early DNS studies of homogeneous isotropic turbulence, ${ }^{22}$ and the $\operatorname{Re}_{\delta}=30,000$ case exceeds the $\operatorname{Re}_{\lambda}=100$ threshold above which turbulence is generally believed to have essentially attained its asymptotic state. ${ }^{2,23}$

\section{B. Probability densities and scaling}

From classical theory, probability densities of the velocity gradients should show widths that scale as $\left(\partial u_{i} / \partial x_{j}\right)_{\mathrm{rms}}$ $\sim\left(\nu / \lambda_{\nu}^{2}\right) \sim\left(u_{c} / \delta\right) \operatorname{Re}_{\delta}^{1 / 2}$. The resulting measured pdfs for each of the on- and off-diagonal gradient component are given in Figs. 2-4 for each of the three combinations of $\operatorname{Re}_{\delta}$ and $\left(S \delta / u_{c}\right)$. Each case is shown in both linear and semilogarithmic axes, which respectively emphasize the central portion and the tails of the pdfs, with normalization on both innerand outer-scale variables. Comparing the two $\left(S \delta / u_{c}\right)=0$ cases at $\operatorname{Re}_{\delta}=6,000$ and 30,000 in Figs. 2 and 3, it is appar- 

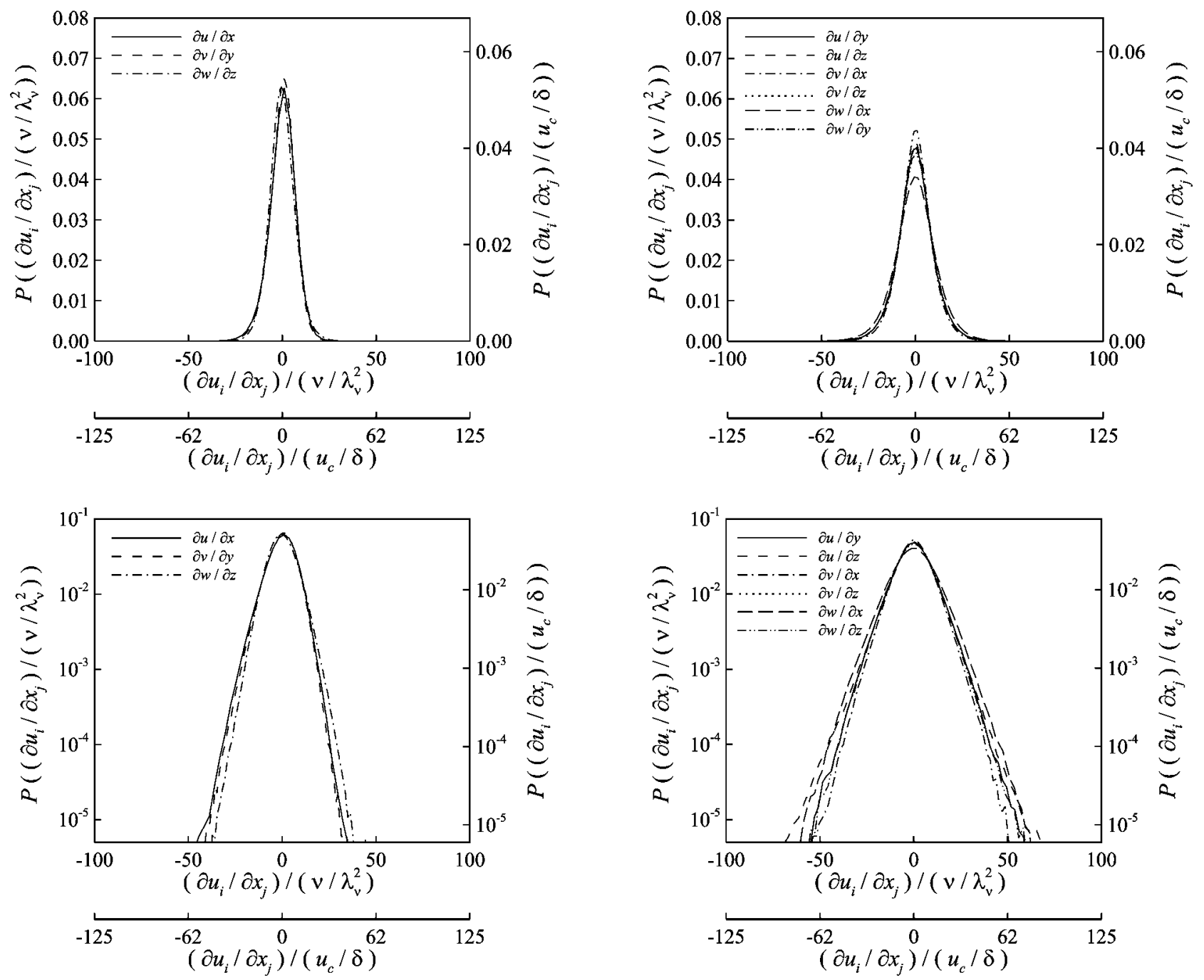

FIG. 3. Probability densities for (left) on-diagonal $(i=j)$ and (right) off-diagonal $(i \neq j)$ components of $\partial u_{i} / \partial x_{j}$ at $\left(\operatorname{Re}_{\delta} ; S\right)=(30,000 ; 0)$ in linear $($ top $)$ and semilogarithmic (bottom) axes scaled on local inner $\left(\nu, \lambda_{\nu}\right)$ and outer $\left(u_{c}, \delta\right)$ variables. Resulting similarity suggests near isotropy in velocity gradient fields.

ent that the relative widths of both sets of corresponding velocity gradient pdfs are essentially identical in the innerscale normalization. Furthermore, consistent with the classical $\operatorname{Re}_{\delta}^{1 / 2}$ scaling above, comparing the axes for the outerscale normalization shows that the corresponding pdfs at $\operatorname{Re}_{\delta}=30,000$ are indeed about $\sqrt{5}$ times wider than at $\operatorname{Re}_{\delta}$ $=6,000$.

\section{Similarity and isotropy}

In incompressible homogeneous isotropic turbulence, the on- and off-diagonal components of the velocity gradients satisfy the similarity and isotropy relation $\overline{\left(\partial u_{i} / \partial x_{j}\right)^{2}}{ }_{i \neq j}$ $=2 \overline{\left(\partial u_{i} / \partial x_{j}\right)^{2}}{ }_{i=j}$. Figure 2 provides tests of this similarity in pdfs of the measured $\partial u_{i} / \partial x_{j}$ values from the most highly resolved case. Isotropy would require the distributions for all three on-diagonal components in Fig. 2 to be identical, and the distributions for the six off-diagonal components in Fig. 2 to also be identical. There is strong evidence for such similarity of the pdfs for each of the velocity gradient components in each panel. The semilogarithmic axes in the lower panel of each figure verify that the similarity demanded by isotropy applies in turbulent shear flows even for relatively rare large-magnitude gradients in the tails of these pdfs, which have a frequency of occurrence nearly $10^{4}$ times smaller than the mean.

Figures 3 and 4 show corresponding similarity and isotropy tests for the $\operatorname{Re}_{\delta}=30,000$ case with $\left(S \delta / u_{c}\right)=0$ and the $\operatorname{Re}_{\delta}=6,000$ case with $\left(S \delta / u_{c}\right)=1.7$. Comparing these with the results above for the $\operatorname{Re}_{\delta}=6,000$ case with $\left(S \delta / u_{c}\right)=0$, it is evident in the pdfs for the on- and off-diagonal components that the two $S=0$ cases in Figs. 2 and 3 provide relatively good agreement with the similarity required by isotropy. In contrast, the $S \neq 0$ case in Fig. 4 shows significant departures from isotropy, as seen by the significantly lower degree of similarity in the pdfs for the off-diagonal velocity gradient components. The pdfs in Fig. 4 for the corresponding on-diagonal components are seen to be very nearly similar and symmetric, while those for the off-diagonal components show significant asymmetry and lack of similarity.

The departures from isotropy in the $\left(S \delta / u_{c}\right)=1.7$ case above are sufficiently large and consistent that they cannot be attributed to the comparatively lower degree of conver- 

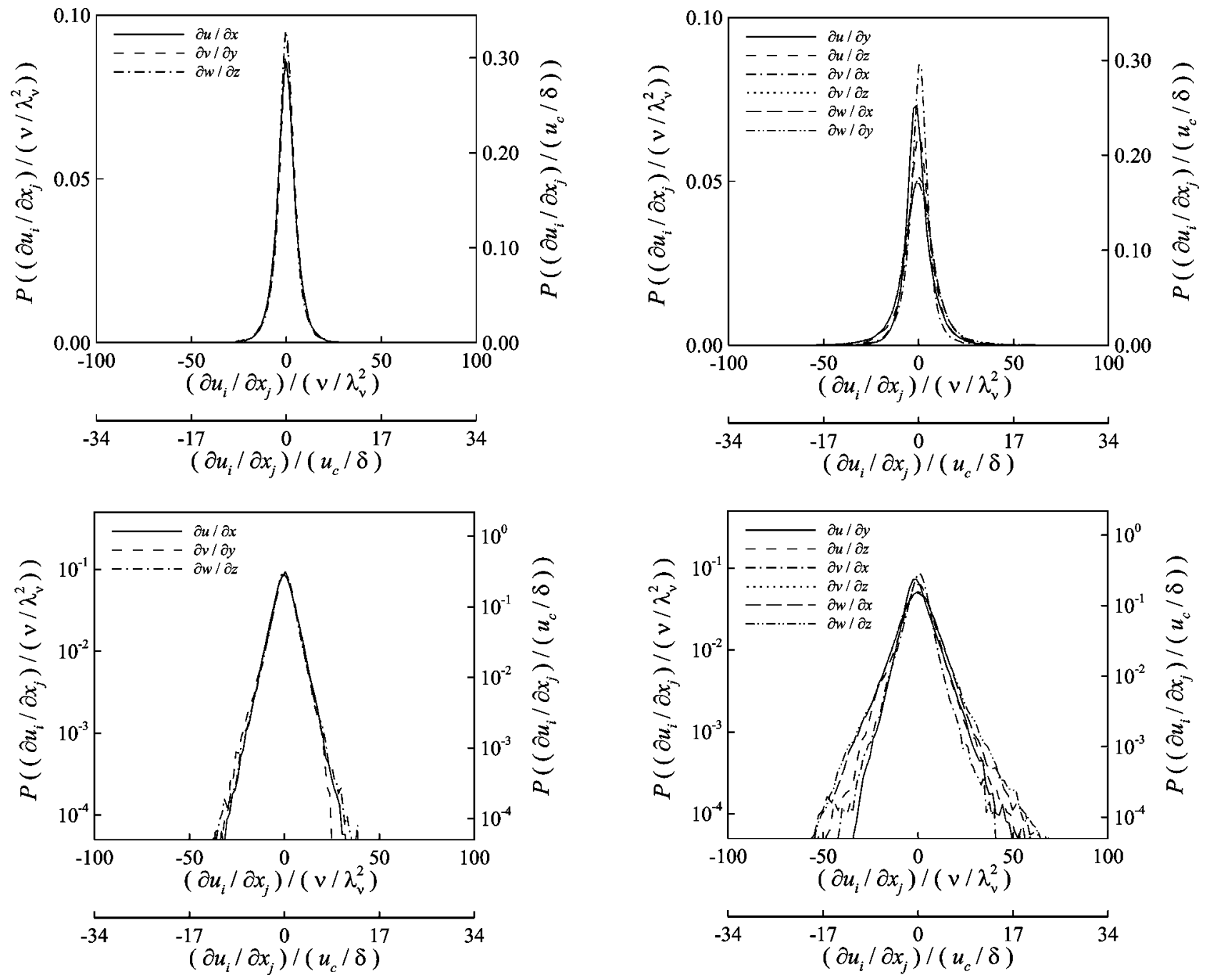

FIG. 4. Probability densities of (left) on-diagonal $(i=j)$ and (right) off-diagonal $(i \neq j)$ components of $\partial u_{i} / \partial x_{j}$ at $\left(\operatorname{Re}_{\delta} ; S\right)=(6,000 ; 1.2)$ in linear $($ top $)$ and semilogarithmic (bottom) axes scaled on local inner $\left(\nu, \lambda_{\nu}\right)$ and outer $\left(u_{c}, \delta\right)$ variables. Departures from strict similarity suggest significant anisotropy in this case.

gence of these distributions. It will be seen in Sec. V that, consistent with previous DNS results for homogeneous uniformly shear turbulence, ${ }^{14}$ the underlying vorticity field for $S \neq 0$ case in this turbulent shear flow shows a strong preferred alignment with the strain rate field imposed by the local mean shear that produces this anisotropy. By contrast, the two $S=0$ cases have no such mean strain field and are seen to agree far more closely with classical results for isotropic turbulence.

Classical theory further suggests, that at sufficiently large $\mathrm{Re}_{\delta}$, velocity gradient distributions of the type in Figs. 2-4 will become independent of $\operatorname{Re}_{\delta}$ when scaled on inner variables. This would require pdfs for the gradient components from the two $S=0$ cases in Figs. 2 and 3 , at $\operatorname{Re}_{\delta}$ $=6,000$ and 30,000, respectively, to be identical in the innerscaled axes. A direct test of this scaling and similarity is shown in Fig. 5, where the inner-scaled distributions for the on- and off-diagonal velocity gradient components are compared. The results show generally good similarity between the $\operatorname{Re}_{\delta}=6,000$ and 30,000 cases for the three on-diagonal components and the six off-diagonal components, and pro- vide further support for the classical isotropy and scaling in the velocity gradient fields for the $S=0$ cases in this turbulent free shear flow.

\section{Moments}

In Figs. 2 and 3 the relative widths of the velocity gradient pdfs for the on- and off-diagonal components in the two $S=0$ cases can be seen to agree reasonably well with the $\sqrt{2}$ ratio in their rms values implied by the second-moment relation from isotropy. Departures from isotropy in all three cases can be quantified by moments of the measured velocity gradient components, given in Tables II-IV. Comparing various combinations of the mean-square gradient values $M_{2}$ with their corresponding isotropic values allows assessments of the degree of anisotropy in each case.

The three combinations shown in Table $\mathrm{V}$ are of particular interest, since they allow various aspects of the velocity gradients to be isolated. The first is based on the ratio of on-diagonal to off-diagonal components of $\partial u_{i} / \partial x_{j}$, for which the isotropic value is $1 / 4$ and the corresponding mea- 

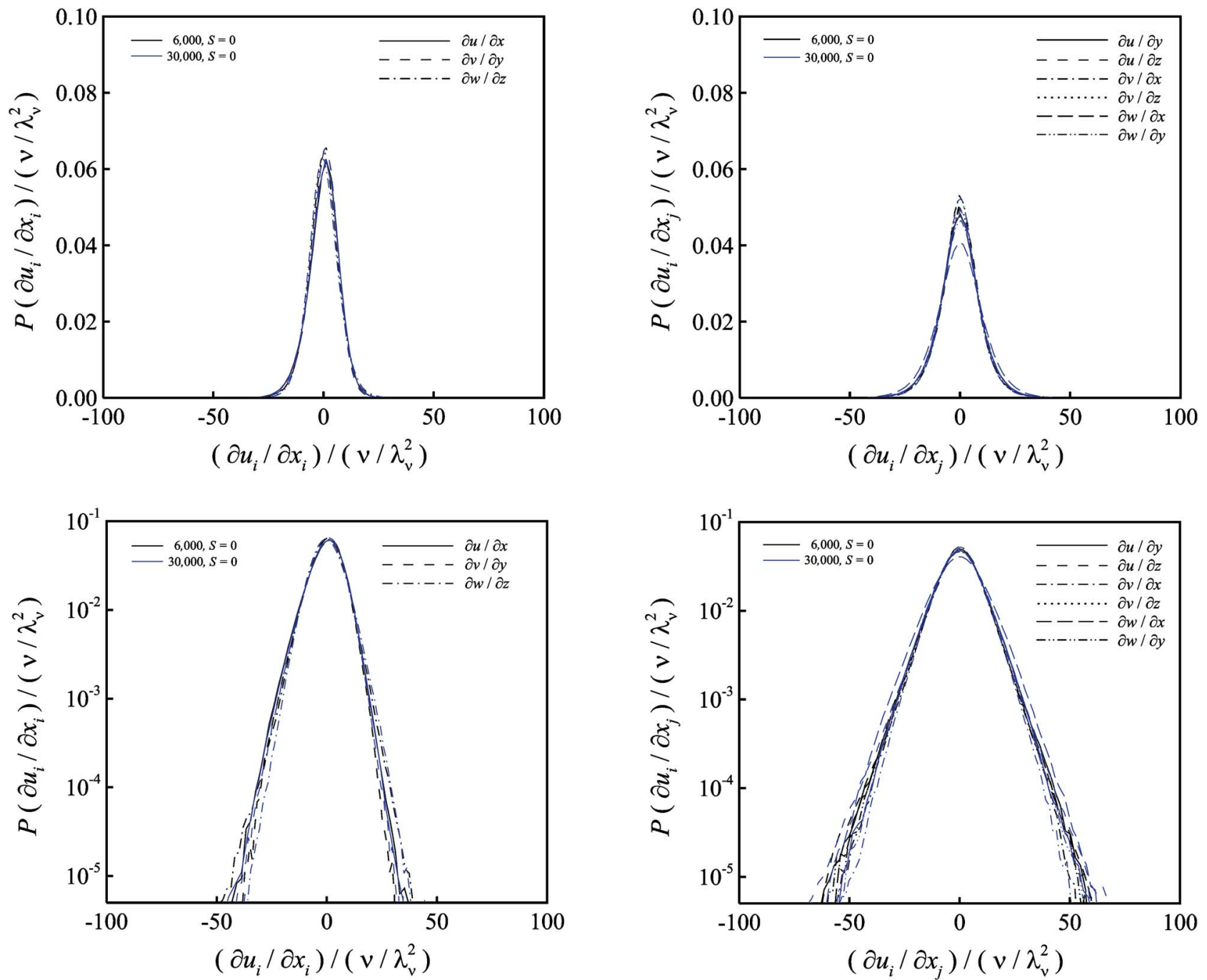

FIG. 5. (Color online) Probability densities of (left) on-diagonal $(i=j)$ and (right) off-diagonal $(i \neq j)$ components of $\partial u_{i} / \partial x_{j}$ for the two $S=0$ cases, in linear (top) and semilogarithmic (bottom) axes, scaled on inner variables $\left(\nu, \lambda_{\nu}\right)$, demonstrating that $\operatorname{Re}_{\delta}$ similarity and isotropy largely account for measured velocity gradients at $\operatorname{Re}_{\delta}=6,000$ and 30,000 .

sured values are 0.26 and 0.24 for the two $S=0$ cases at $\operatorname{Re}_{\delta}=6,000$ and 30,000, and 0.21 for the $S \neq 0$ case at $\operatorname{Re}_{\delta}$ $=6,000$. A second combination is based the ratio of $z$ derivatives to $x$ and $y$ derivatives. In this case the isotropic value is

TABLE III. Moment values scaled on inner variables $\left(\nu, \lambda_{\nu}\right)$ for all nine components of the velocity gradient field $\partial u_{i} / \partial x_{j}$ measured at $\left(\operatorname{Re}_{\delta} ; S\right)$ $=(30,000 ; 0)$, for which $\operatorname{Re}_{\lambda}=113$, showing mean $M_{1}$, rms fluctuation $M_{2}$, skewness $M_{3}$, and kurtosis $M_{4}$.

\begin{tabular}{ccccc}
\hline \hline $\begin{array}{c}\text { Derivative } \\
\text { component }\end{array}$ & $M_{1}$ & $M_{2}$ & $M_{3}$ & $M_{4}$ \\
\hline$\partial u / \partial x$ & -0.006 & 7.065 & -0.355 & 4.808 \\
$\partial u / \partial y$ & -0.003 & 9.817 & 0.021 & 4.404 \\
$\partial u / \partial z$ & 0.000 & 9.968 & -0.065 & 5.503 \\
$\partial v / \partial x$ & 0.004 & 9.030 & 0.006 & 4.291 \\
$\partial v / \partial y$ & -0.004 & 6.779 & -0.345 & 3.820 \\
$\partial v / \partial z$ & -0.004 & 9.967 & -0.060 & 5.497 \\
$\partial w / \partial x$ & -0.000 & 11.228 & -0.012 & 4.107 \\
$\partial w / \partial y$ & 0.004 & 9.800 & 0.003 & 4.211 \\
$\partial w / \partial z$ & -0.006 & 6.922 & 0.136 & 3.742 \\
\hline \hline
\end{tabular}

$1 / 2$, and the respective measured values are 0.50 and 0.50 for the two $S=0$ cases at $\operatorname{Re}_{\delta}=6,000$ and 30,000, and 0.48 for the $S \neq 0$ case at $\operatorname{Re}_{\delta}=6,000$. A final combination is based on the ratio of $w$ gradients to $u$ and $v$ gradients, for which the

TABLE IV. Moment values scaled on inner variables $\left(\nu, \lambda_{\nu}\right)$ for all nine components of the velocity gradient field $\partial u_{i} / \partial x_{j}$ measured at $\left(\operatorname{Re}_{\delta} ; S\right)$ $=(6,000 ; 1.2)$, for which $\operatorname{Re}_{\lambda}=43$, showing mean $M_{1}$, rms fluctuation $M_{2}$, skewness $M_{3}$, and kurtosis $M_{4}$.

\begin{tabular}{ccccc}
\hline \hline $\begin{array}{c}\text { Derivative } \\
\text { component }\end{array}$ & $M_{1}$ & $M_{2}$ & $M_{3}$ & $M_{4}$ \\
\hline$\partial u / \partial x$ & -0.036 & 6.091 & 0.069 & 5.846 \\
$\partial u / \partial y$ & 0.023 & 8.024 & 1.122 & 9.712 \\
$\partial u / \partial z$ & -0.006 & 9.160 & 0.015 & 8.362 \\
$\partial v / \partial x$ & -0.013 & 7.016 & -0.223 & 7.567 \\
$\partial v / \partial y$ & -0.043 & 6.025 & -0.499 & 5.869 \\
$\partial v / \partial z$ & -0.008 & 9.168 & -0.125 & 7.619 \\
$\partial w / \partial x$ & -0.014 & 10.588 & 0.073 & 7.602 \\
$\partial w / \partial y$ & 0.004 & 11.081 & 0.234 & 7.600 \\
$\partial w / \partial z$ & -0.041 & 6.023 & -0.024 & 7.032 \\
\hline \hline
\end{tabular}


TABLE V. Measured values for various combinations of mean-square velocity gradient ratios for all three cases, showing corresponding isotropic values (right) for comparison.

\begin{tabular}{|c|c|c|c|c|}
\hline & $\operatorname{Re}_{\delta}=6,000$ & $\operatorname{Re}_{\delta}=30,000$ & $\operatorname{Re}_{\delta}=6,000$ & \\
\hline Validation component & $\begin{aligned} \operatorname{Re}_{\lambda} & =45 \\
S & =0\end{aligned}$ & $\begin{aligned} \operatorname{Re}_{\lambda} & =113 \\
S & =0\end{aligned}$ & $\begin{array}{c}\operatorname{Re}_{\lambda}=43 \\
S=1.2\end{array}$ & $\begin{array}{l}\text { Isotropic } \\
\text { value }\end{array}$ \\
\hline$\overline{\left(\frac{\partial u}{\partial x}\right)^{2}}+\overline{\left(\frac{\partial v}{\partial y}\right)^{2}}+\overline{\left(\frac{\partial w}{\partial z}\right)^{2}}$ & \multirow[b]{2}{*}{0.26} & \multirow[b]{2}{*}{0.24} & \multirow[b]{2}{*}{0.21} & \multirow[b]{2}{*}{0.25} \\
\hline 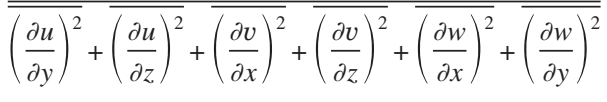 & & & & \\
\hline$\overline{\left(\frac{\partial w}{\partial x}\right)^{2}}+\overline{\left(\frac{\partial w}{\partial y}\right)^{2}}+\overline{\left(\frac{\partial w}{\partial z}\right)^{2}}$ & \multirow[b]{2}{*}{0.51} & \multirow[b]{2}{*}{0.57} & \multirow[b]{2}{*}{0.76} & \multirow[b]{2}{*}{0.50} \\
\hline 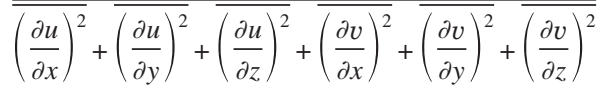 & & & & \\
\hline$\overline{\left(\frac{\partial u}{\partial z}\right)^{2}}+\overline{\left(\frac{\partial v}{\partial z}\right)^{2}}+\overline{\left(\frac{\partial w}{\partial z}\right)^{2}}$ & \multirow[b]{2}{*}{0.50} & \multirow[b]{2}{*}{0.50} & \multirow[b]{2}{*}{0.48} & \multirow[b]{2}{*}{0.50} \\
\hline$\overline{\overline{\left(\frac{\partial u}{\partial x}\right)^{2}}+\overline{\left(\frac{\partial u}{\partial y}\right)^{2}}+\overline{\left(\frac{\partial v}{\partial x}\right)^{2}}+\overline{\left(\frac{\partial v}{\partial y}\right)^{2}}+\overline{\left(\frac{\partial w}{\partial x}\right)^{2}}+\overline{\left(\frac{\partial w}{\partial y}\right)^{2}}}$ & & & & \\
\hline
\end{tabular}

isotropic value is $1 / 2$. The corresponding measured values are 0.51 and 0.57 , respectively, for the $S=0$ cases at $\operatorname{Re}_{\delta}$ $=6,000$ and 30,000, and 0.76 for the $S \neq 0$ case at $\operatorname{Re}_{\delta}$ $=6,000$. Collectively, these moment ratios provide further support for the observation that velocity gradient fields from the two $S=0$ cases in this turbulent shear flow are largely isotropic, and that the $\left(S \delta / u_{c}\right)=1.7$ case shows significantly greater anisotropy.

\section{E. Comparisons with homogeneous isotropic turbulence}

Table VI compares the measured values for the thirdand fourth-moments of the longitudinal velocity gradients from the present turbulent shear flow for the two $S=0$ cases at $\operatorname{Re}_{\lambda}=45$ and 113 with corresponding results from DNS of homogeneous isotropic turbulence at $\operatorname{Re}_{\lambda}=54$ and 125 from Ref. 23 and $\operatorname{Re}_{\lambda}=61$ and 168 from Ref. 22. Although the $\operatorname{Re}_{\lambda}$

TABLE VI. Comparisons of present measured velocity gradient moment values $M_{3}$ and $M_{4}$ with corresponding results from DNS studies of homogeneous isotropic turbulence at comparable $\operatorname{Re}_{\lambda}$ values.

\begin{tabular}{cccc}
\hline \hline Component & $\begin{array}{c}\text { Present } \\
\text { data }\end{array}$ & $\begin{array}{c}\text { Gotoh } \\
\text { et al. }\end{array}$ & $\begin{array}{c}\text { Jimenez } \\
\text { et al. }\end{array}$ \\
\hline $\operatorname{Re}_{\lambda}$ & 45 & 54 & 61 \\
$M_{3}(\partial u / \partial x)$ & -0.428 & -0.517 & -0.495 \\
$M_{4}(\partial u / \partial x)$ & 4.22 & 4.47 & 4.60 \\
$\operatorname{Re}_{\lambda}$ & 113 & 125 & 168 \\
$M_{3}(\partial u / \partial x)$ & -0.355 & -0.529 & -0.525 \\
$M_{4}(\partial u / \partial x)$ & 4.81 & 5.65 & 6.10 \\
\hline \hline
\end{tabular}

values are comparable, the present results are from a turbulent free shear flow and thus differences relative to these numerical studies of homogeneous isotropic turbulence may be expected. Nevertheless, the $M_{4}$ values follow a consistent trend with increasing $\operatorname{Re}_{\lambda}$ in the measured results and in the DNS results, suggesting relatively little difference in the flatness between isotropic and shear flow turbulence. However for the $M_{3}$ values there is no such consistent trend seen in these results between isotropic and shear flow turbulence. Moreover, there are significant differences apparent in the $\operatorname{Re}_{\lambda}$ trend even between the two numerical studies. Table VII thus makes a further comparison of the present measured $M_{3}$ values from the present turbulent shear flow at $\operatorname{Re}_{\lambda}=45$ with values obtained experimentally ${ }^{24}$ in grid turbulence at $\operatorname{Re}_{\lambda}$ $=52$ using multiple hot-wire probes. The three longitudinal velocity gradients are shown in order of decreasing $M_{3}$ values. There is generally good agreement between the two sets of results, suggesting that the differences in Table VI may be real.

TABLE VII. Comparisons of present measured velocity gradient moment values $M_{3}$ with corresponding results from hot-wire measurements in grid turbulence of Tsinober et al. at comparable $\operatorname{Re}_{\lambda}$.

\begin{tabular}{ccc}
\hline \hline Component & $\begin{array}{c}\text { Present } \\
\text { data }\end{array}$ & $\begin{array}{c}\text { Tsinober } \\
\text { et al. }\end{array}$ \\
\hline $\operatorname{Re}_{\lambda}$ & 45 & 52 \\
$M_{3}(\partial u / \partial x)$ & -0.43 & -0.50 \\
$M_{3}(\partial v / \partial y)$ & -0.38 & -0.37 \\
$M_{3}(\partial w / \partial z)$ & -0.14 & -0.14 \\
\hline \hline
\end{tabular}



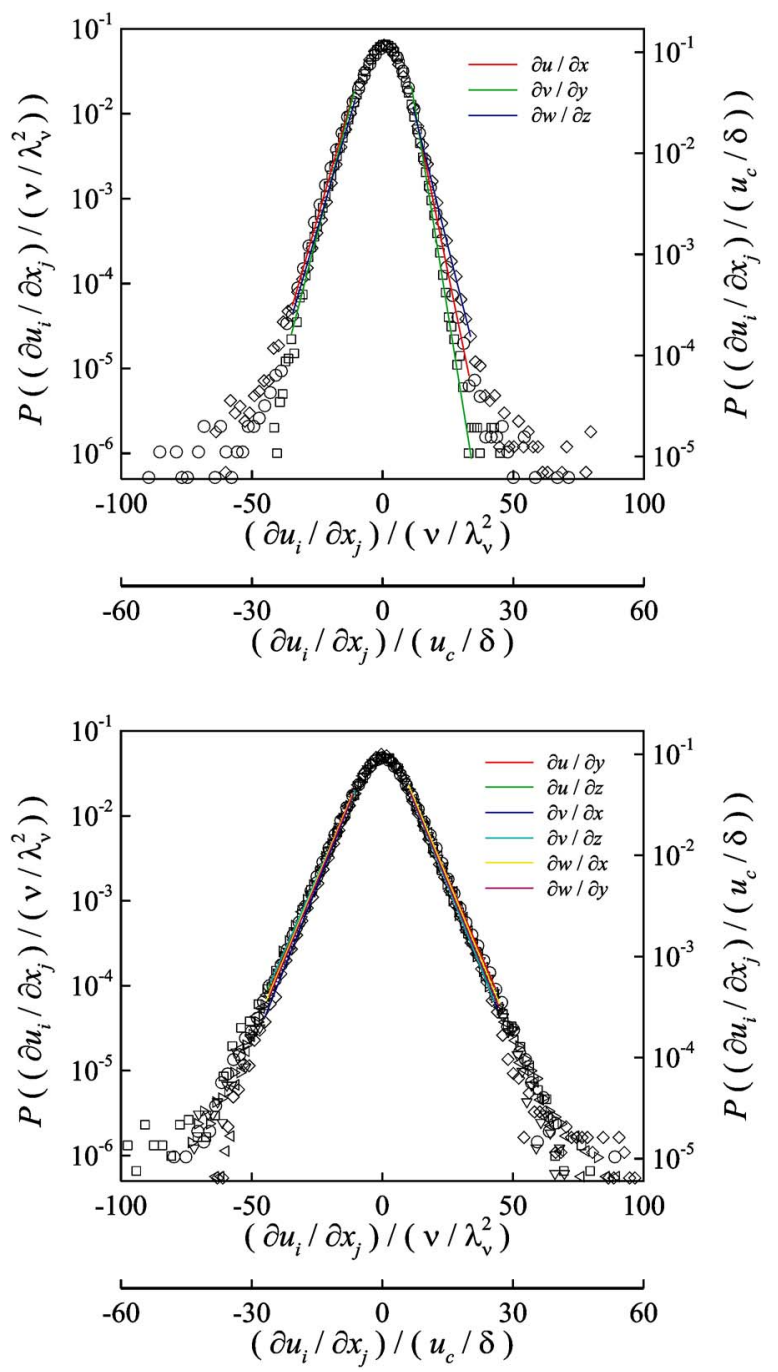

FIG. 6. (Color online) Least-squares fits to exponential tails of probability densities of $\partial u_{i} / \partial x_{j}$ for $i=j$ (top) and $i \neq j$ (bottom) from measured velocity gradients at $\operatorname{Re}_{\delta}=6,000, S=0$. Resulting scaling constants $\alpha \equiv-(\mathrm{d} / \mathrm{d}|q|) \log P(q)$ for left and right tails of each component $q$ are given in Table VIII.

\section{F. Exponential scaling of the pdf tails}

The semilogarithmic forms in Figs. 2-4 show strong evidence for exponential scaling of the form $P(q) \sim e^{-\alpha|q|}$ at large values of $|q|$ in the tails of these distributions, corresponding to relatively rare occurrences of large positive and negative gradient values. The scaling constant $\alpha$ in each of the tails can thus be obtained as

$$
\alpha=\frac{-d}{d|q|} \log P(q) .
$$

The complete set of these scaling constants quantifies the relative occurrence of such large positive and negative values in each of the gradient component fields.

Figure 6 shows linear least-squares fits to the left and right tails of each of the velocity gradient pdfs for the most highly resolved case. It has been known for some time from studies of velocity gradients in Burgers turbulence that the two tails can have different exponential scaling constants. ${ }^{25}$ Similar observations have been made in velocity gradient pdfs from DNS studies of homogeneous isotropic or uniformly sheared turbulence. ${ }^{23}$ It is apparent in Fig. 6 that in shear flow turbulence the scaling of the left and right tails is also significantly different, especially for the on-diagonal components of $\left(\partial u_{i} / \partial x_{j}\right)$. Table IV gives the resulting leftand right-tail $\alpha$ values for all three cases from (1). It can be seen that, even for the two $S=0$ cases, there are substantial differences in the tail-scaling constants among the ondiagonal (or off-diagonal) velocity gradient components. Such differences would represent further relatively weak departures from isotropy, since they correspond to differing frequencies of occurrence of relatively rare positive and negative large-gradient values for the various components (see Table VIII).

\section{STRAIN RATE FIELDS}

Section III revealed large departures from isotropy in velocity gradient fields for the $S \neq 0$ case, and weaker departures for the two $S=0$ cases. This section examines the mani-

TABLE VIII. Results from present study for scaling constants $\alpha \equiv-(\mathrm{d} / \mathrm{d}|q|) \log P(q)$ in left and right exponential tails from least-squares fits to the inner-scaled probability densities of velocity gradient components $\partial u_{i} / \partial x_{j}$ in Fig. 6.

\begin{tabular}{|c|c|c|c|c|c|c|}
\hline \multirow[b]{2}{*}{$\begin{array}{l}\text { Gradient } \\
\text { component }\end{array}$} & \multicolumn{3}{|c|}{ Left-tail scaling exponent $\alpha$} & \multicolumn{3}{|c|}{ Right-tail scaling exponent $\alpha$} \\
\hline & $\begin{array}{c}\operatorname{Re}_{\delta}=6,000 \\
\operatorname{Re}_{\lambda}=45 \\
S=0\end{array}$ & $\begin{aligned} \operatorname{Re}_{\delta} & =30,000 \\
\operatorname{Re}_{\lambda} & =113 \\
S & =0\end{aligned}$ & $\begin{array}{c}\operatorname{Re}_{\delta}=6,000 \\
\operatorname{Re}_{\lambda}=43 \\
S=1.2\end{array}$ & $\begin{array}{c}\operatorname{Re}_{\delta}=6,000 \\
\operatorname{Re}_{\lambda}=45 \\
S=0\end{array}$ & $\begin{aligned} \operatorname{Re}_{\delta} & =30,000 \\
\operatorname{Re}_{\lambda} & =113 \\
S & =0\end{aligned}$ & $\begin{array}{c}\operatorname{Re}_{\delta}=6,000 \\
\operatorname{Re}_{\lambda}=43 \\
S=1.2\end{array}$ \\
\hline$\partial u / \partial x$ & 0.24 & 0.24 & 0.24 & 0.34 & 0.35 & 0.25 \\
\hline$\partial v / \partial y$ & 0.27 & 0.26 & 0.22 & 0.44 & 0.37 & 0.42 \\
\hline$\partial w / \partial z$ & 0.24 & 0.31 & 0.20 & 0.36 & 0.35 & 0.20 \\
\hline$\partial u / \partial y$ & 0.16 & 0.16 & 0.20 & 0.16 & 0.17 & 0.20 \\
\hline$\partial u / \partial z$ & 0.16 & 0.16 & 0.15 & 0.16 & 0.17 & 0.21 \\
\hline$\partial v / \partial x$ & 0.17 & 0.19 & 0.18 & 0.17 & 0.19 & 0.15 \\
\hline$\partial v / \partial z$ & 0.15 & 0.15 & 0.13 & 0.39 & 0.33 & 0.33 \\
\hline$\partial w / \partial x$ & 0.15 & 0.13 & 0.12 & 0.38 & 0.36 & 0.31 \\
\hline$\partial w / \partial y$ & 0.16 & 0.16 & 0.12 & 0.37 & 0.32 & 0.30 \\
\hline
\end{tabular}




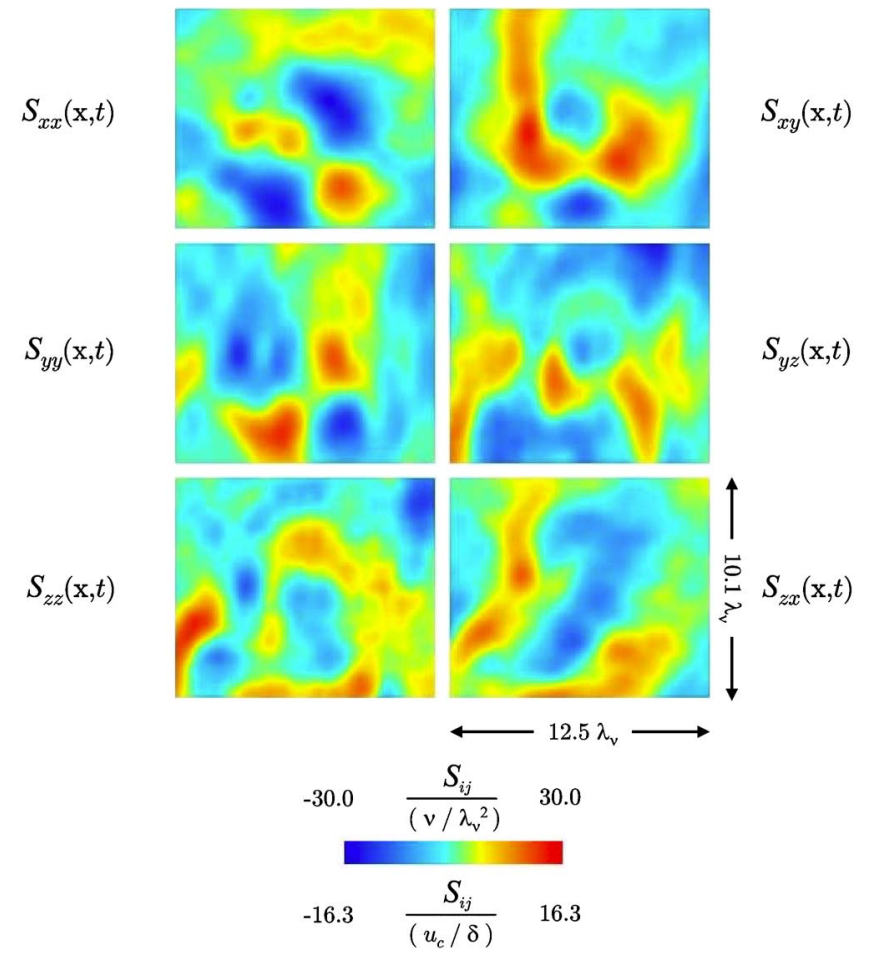

FIG. 7. (Color online) Typical six-component strain tensor field $S_{i j}(\mathbf{x}, t)$ at $\left(\operatorname{Re}_{\delta} ; S\right)=(6,000 ; 0)$. Field of view is shown normalized by local inner length scale $\lambda_{v}$. Color scale shows quantitative values normalized on local inner $\left(\nu, \lambda_{\nu}\right)$ and outer $\left(u_{c}, \delta\right)$ scales.

festation of these departures in the strain rate field

$$
S_{i j}(\mathbf{x}, t) \equiv\left(\frac{\partial u_{i}}{\partial x_{j}}+\frac{\partial u_{j}}{\partial x_{i}}\right),
$$

which drives the underlying vorticity field in Sec. V. Figure 7 gives a typical example of the instantaneous strain rate tensor component fields, corresponding to the same data plane as other fields shown in following sections to provide an indication of the relative structures in each of these fields.

Resulting measured distributions of the normal and shear strain rate components for each of the three cases are shown in Figs. 8-10. For the two $S=0$ cases at $\operatorname{Re}_{\delta}=6,000$ and 30,000 these show generally good agreement with the similarity implied by isotropy. For the $S \neq 0$ case it is apparent that the normal strain rate components in Fig. 10 show comparably good similarity as seen for the other two cases, however the shear components in Fig. 10 show very significant departures from similarity. This lack of similarity cannot be attributed to the smaller number of data planes for that case, since the corresponding normal components show good agreement with similarity.

A further test of both the scaling and similarity in the strain rate component fields is shown in Fig. 11, where the measured component values from the two $S=0$ cases at $\operatorname{Re}_{\delta}=6,000$ and 30,000 are compared. It is apparent that when scaled on inner variables the strain rate values at $S$ $=0$ in this turbulent shear flow are essentially identical, in conformance with the scaling and isotropy implied by clas-
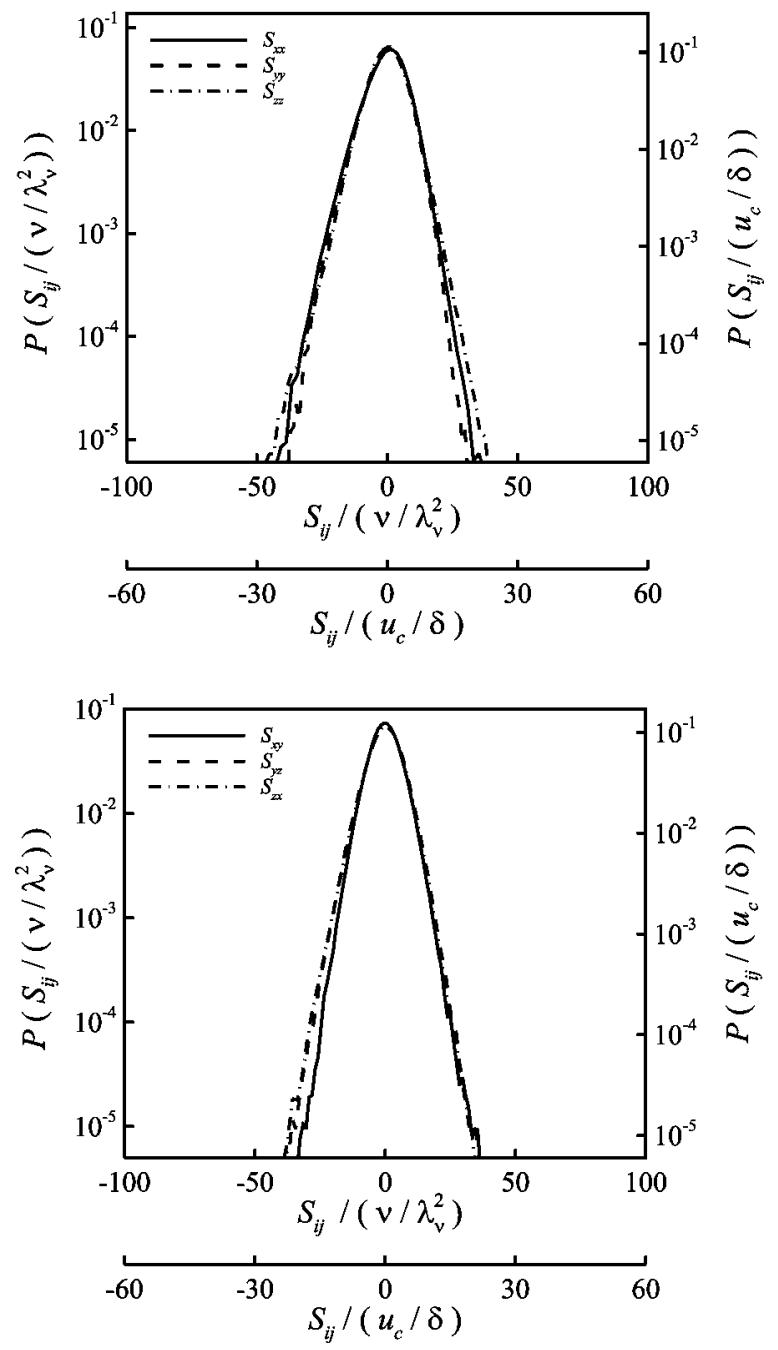

FIG. 8. Probability densities of on-diagonal (top) and off-diagonal (bottom) components of the strain rate fields $S_{i j}(\mathbf{x}, t)$ at $\left(\operatorname{Re}_{\delta} ; S\right)=(6,000 ; 0)$, scaled on local inner $\left(\nu, \lambda_{\nu}\right)$ and outer $\left(u_{c}, \delta\right)$ variables.

sical theory. Moreover, many of the relatively weak departures from isotropy can be seen in these distributions to be largely similar at both $\operatorname{Re}_{\delta}$ values.

\section{A. Principal strain rates}

The eigenvalues $S_{i}$ of the local strain rate tensor play a key role in the local structure and dynamics of the vorticity field. Figure 12 gives the probability densities of the three principal strain rates $S_{1}, S_{2}$, and $S_{3}$ for each case, where in the notation used here $S_{1} \geqslant S_{2} \geqslant S_{3}$. Incompressibility requires $S_{1}+S_{2}+S_{3}=0$, and thus $S_{1} \geqslant 0$ and $S_{3} \leqslant 0$. In solving for the principal strain rates from the measured velocity gradients, any points that fail to meet the latter two criteria are rejected, since these represent instances where small measurement errors lead to nonphysical combinations of the velocity gradients. Note that less than $0.6 \%$ of all points failed to meet these criteria, reflecting the high accuracy of these DSPIV measurements. The distributions in Fig. 12 can also be compared with Fig. $5 c$ of Ref. 26, which are in good agreement with the present results for the two $S=0$ cases.

Consistent with classical scaling, the principal strain rate 

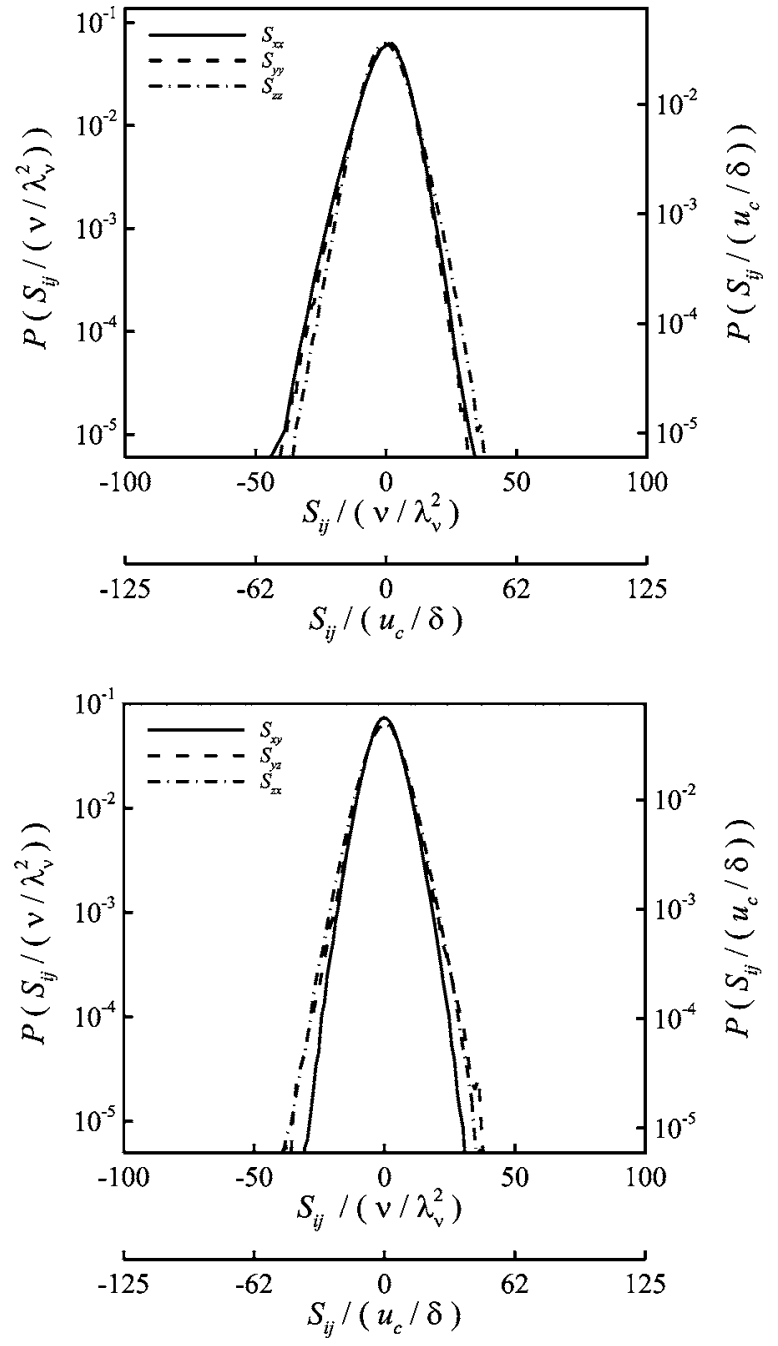

FIG. 9. Probability densities of on-diagonal (top) and off-diagonal (bottom) components of the strain rate fields $S_{i j}(\mathbf{x}, t)$ at $\left(\operatorname{Re}_{\delta} ; S\right)=(30,000 ; 0)$, scaled on local inner $\left(\nu, \lambda_{\nu}\right)$ and outer $\left(u_{c}, \delta\right)$ variables.

distributions for the two $S=0$ cases at $\operatorname{Re}_{\delta}=6,000$ and 30,000 , when scaled with the inner-scale strain rate $\left(\nu / \lambda_{\nu}^{2}\right)$ as in Fig. 12, can be seen to be nearly identical. The corresponding outer-scaling $S_{i} \sim\left(u_{c} / \delta\right) \operatorname{Re}_{\delta}^{1 / 2}$ is confirmed in Table IX, where the proportionality constants in both the inner- and outer-variable scalings for the mean $S_{i}$ values are seen to be essentially identical for the two $S=0$ cases. However for the $S \neq 0$ case Fig. 12 shows a substantial difference, especially in the $S_{1}$ distribution.

Various parameters have been used to characterize the relative magnitudes of the three principal strain rates. ${ }^{27}$ Here we use the ratio $\sigma \equiv S_{2} / S_{1}$, which from incompressibility is bounded by $-1 / 2 \leqslant \sigma \leqslant 1$. The local $\sigma$ value plays a key role in the structure and dynamics of gradients fields in turbulent flows. ${ }^{5,6}$ In particular, where $\sigma<0$ the local strain field tends toward formation of line-like concentrations of vorticity, while for $\sigma>0$ the strain field promotes development of sheet-like structures. Figure 13 shows distributions of the measured $\sigma$ values for all three cases. Note that less than $6 \%$ of all points produced $\sigma$ value outside the range allowed by incompressibility, consistent with minimum limits of divergence errors in DSPIV measurements obtained in Ref. 18.
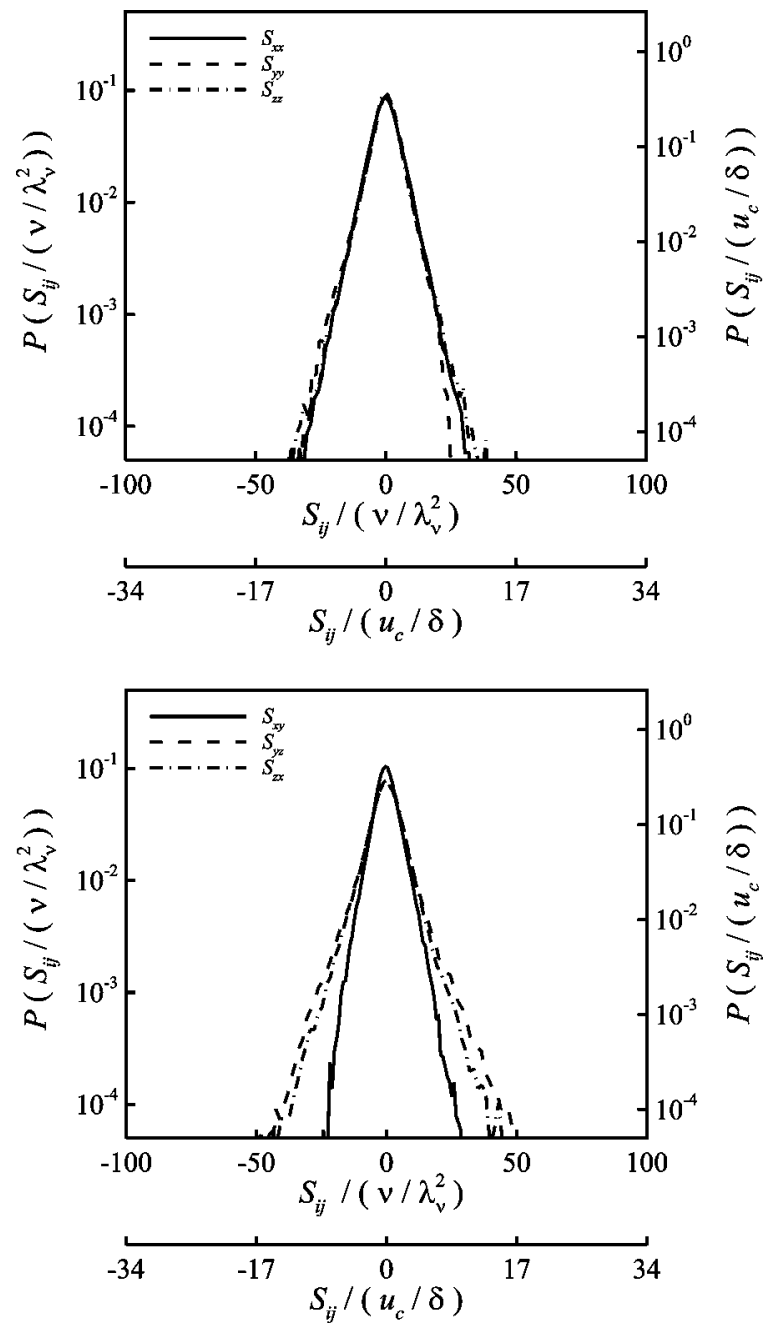

FIG. 10. Probability densities of on-diagonal (top) and off-diagonal (bottom) components of the strain rate fields $S_{i j}(\mathbf{x}, t)$ at $\left(\operatorname{Re}_{\delta} ; S\right)=(6,000 ; 1.2)$, scaled on local inner $\left(\nu, \lambda_{\nu}\right)$ and outer $\left(u_{c}, \delta\right)$ variables. Results show similarity in on-diagonal components, but strong departures from similarity in off-diagonal components.

The shapes of the $\sigma$ distributions for the two $S=0$ cases are largely similar, while the $S \neq 0$ case shows an increased tendency of the flow to form line-like vortical structures.

\section{B. Eigenvector alignments}

For each of the three cases, Figs. 14 and 15 show the measured distributions of the two spherical orientation angles $\varphi$ and $\vartheta$ that give the alignment of the three strain rate eigenvectors $\hat{e}_{i}$. Here $\varphi$ is the polar angle measured from the mean streamwise direction and $\vartheta$ is the azimuthal angle in the transverse plane. In the absence of any preferred eigenvector orientation, spherical symmetry would require the isotropic joint distribution

$$
P(\vartheta, \varphi)=\frac{1}{4 \pi} \sin \varphi
$$

with the corresponding isotropic marginal distributions 

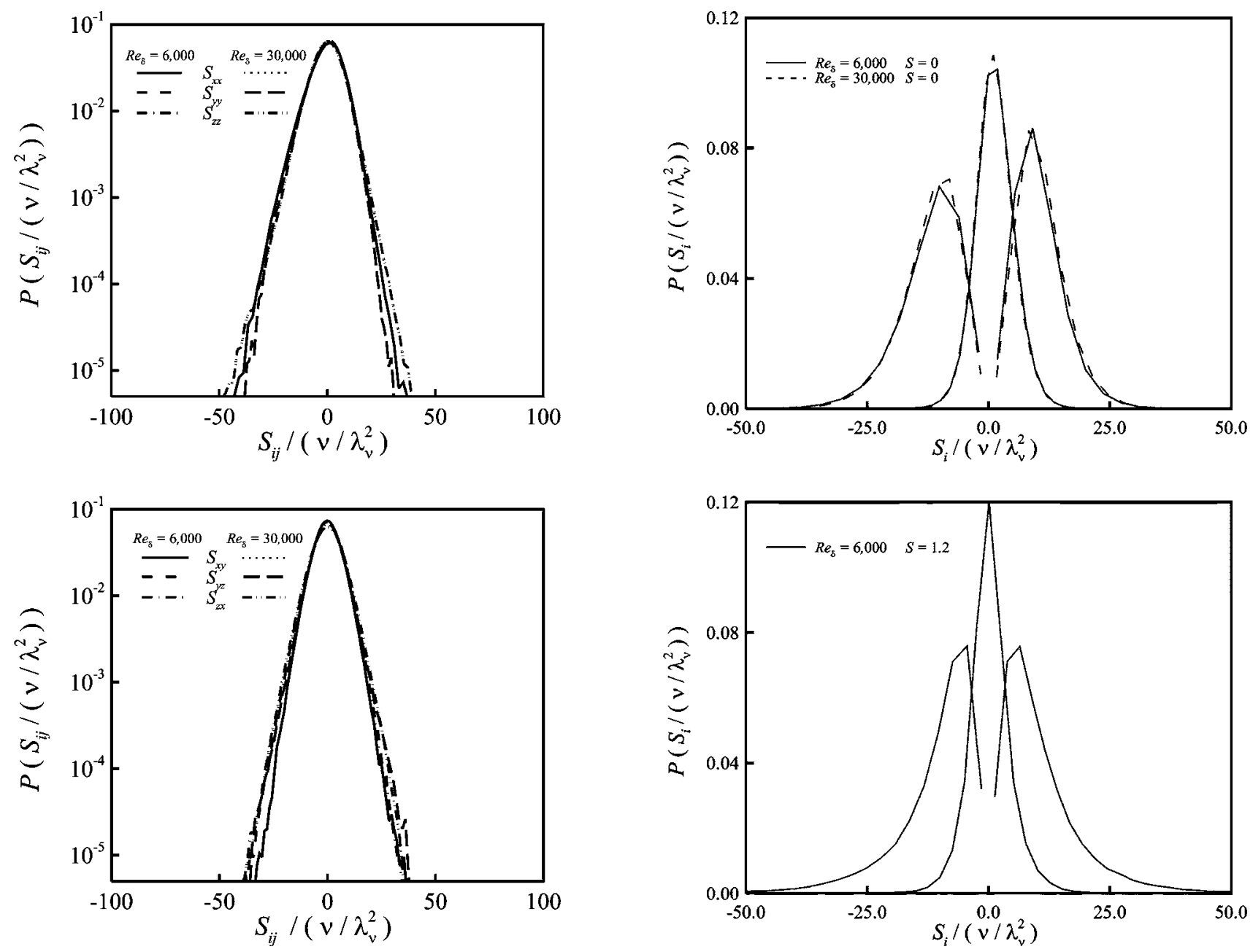

FIG. 11. Probability densities of on-diagonal (top) and off-diagonal (bottom) components of strain rate fields $S_{i j}(\mathbf{x}, t)$ for the two $S=0$ cases at $\operatorname{Re}_{\delta}=6,000$ and 30,000 , scaled on inner variables $\left(\nu, \lambda_{\nu}\right)$ to assess $\operatorname{Re}_{\delta}$ similarity and isotropy in measured strain rate fields.

$$
P(\vartheta)=\frac{1}{2 \pi} \text { and } P(\varphi)=\frac{1}{2} \sin \varphi .
$$

Each of these isotropic marginal distributions is shown by the solid lines in Figs. 14 and 15.

The $\varphi$ distributions for the two $S=0$ cases in Figs. 14 (top, middle) are very similar, suggesting that the departures from strict isotropy in each of these figures are not due to lack of statistical convergence, but are signatures of weak anisotropy in the strain rate fields. As noted in Sec. II, even for the $S=0$ cases there is a relatively weak mean shear on either side of the flow centerline over the field of view of the measurements. This is further supported by the $\varphi$ distribution for the $S \neq 0$ case in Fig. 14 (bottom), which has a largely similar shape as in the two $S=0$ cases, but has higher peaks corresponding to the larger departures from isotropy created by the local mean shear. The two peaks in Fig. 14 (bottom) near $\varphi=\pi / 4$ and $3 \pi / 4$ correspond to the most extensional principal axes of the strain rate field imposed by the mean flow, further indicating that these departures from isotropy are due to the mean shear, even in the two nominally $S=0$ cases.

FIG. 12. Probability densities of principal strain rates $\left(S_{1}, S_{2}, S_{3}\right)$, corresponding to local eigenvalues of the strain rate tensor fields $S_{i j}(\mathbf{x}, t)$, for the $S=0$ cases at $\operatorname{Re}_{\delta}=6,000$ and 30,000 (top), and for $S \neq 0$ at $\operatorname{Re}_{\delta}=6,000$ (bottom), shown scaled on inner variables to test $\operatorname{Re}_{\delta}$ similarity.

Since there is no preferred $\vartheta$ alignment due to the mean shear, the $\vartheta$ distributions for all three cases in Fig. 15 show no consistent patterns. The deviations from isotropy are thus largely attributable to statistical convergence in these distributions.

\section{VORTICITY FIELDS}

This section examines the effect of the outer-scale Reynolds number $\operatorname{Re}_{\delta}$ and the mean shear rate $S$ on the vorticity field

$$
\omega_{k}(\mathbf{x}, t) \equiv \frac{1}{2}\left(\frac{\partial u_{j}}{\partial x_{i}}-\frac{\partial u_{i}}{\partial x_{j}}\right) \varepsilon_{i j k}
$$

in shear flow turbulence. Figure 16 shows the instantaneous vorticity vector components in the same data plane as the fields shown for $\operatorname{Re}_{\delta}=6,000$ and $S=0$ in other sections. Measured distributions of the vorticity components $\omega_{i}$ for each of the three cases are shown in Fig. 17. It is apparent that for the two $S=0$ cases the vorticity field is very nearly isotropic. However the inner-scaled pdfs for the $S \neq 0$ case are fundamentally different from these, reflecting a clear effect of the 
TABLE IX. Scaling constants for scaling laws in outer and inner variables.

\begin{tabular}{|c|c|c|c|c|c|c|c|c|}
\hline \multirow[b]{3}{*}{$\begin{array}{l}\text { Scaling } \\
\text { constant }\end{array}$} & \multicolumn{4}{|c|}{ Outer scale normalization } & \multicolumn{4}{|c|}{ Inner scale normalization } \\
\hline & & $\operatorname{Re}_{\delta}=6,000$ & $\operatorname{Re}_{\delta}=30,000$ & $\operatorname{Re}_{\delta}=6,000$ & & $\operatorname{Re}_{\delta}=6,000$ & $\operatorname{Re}_{\delta}=30,000$ & $\operatorname{Re}_{\delta}=6,000$ \\
\hline & $\begin{array}{l}\text { Scaling } \\
\text { law }\end{array}$ & $\begin{array}{c}\operatorname{Re}_{\lambda}=45 \\
S=0\end{array}$ & $\begin{aligned} \operatorname{Re}_{\lambda} & =113 \\
S & =0\end{aligned}$ & $\begin{array}{c}\operatorname{Re}_{\lambda}=43 \\
S=1.2\end{array}$ & $\begin{array}{c}\text { Scaling } \\
\text { law }\end{array}$ & $\begin{array}{c}\operatorname{Re}_{\lambda}=45 \\
S=0\end{array}$ & $\begin{aligned} \operatorname{Re}_{\lambda} & =113 \\
S & =0\end{aligned}$ & $\begin{array}{c}\operatorname{Re}_{\lambda}=43 \\
S=1.2\end{array}$ \\
\hline$C_{f}$ & $\lambda_{f} / \delta \mathrm{Re}_{\delta}^{-1 / 2}$ & 3.63 & 3.63 & 5.54 & & $\cdots$ & $\cdots$ & $\cdots$ \\
\hline$C_{S 1}$ & $\overline{S_{1}} /\left(u_{c} / \delta\right) \operatorname{Re}_{\delta}^{1 / 2}$ & 0.077 & 0.079 & 0.039 & $\overline{S_{1}} /\left(\nu / \lambda_{\nu}^{2}\right)$ & 11.1 & 11.5 & 10.6 \\
\hline$C_{S 2}$ & $\overline{S_{2}} /\left(u_{c} / \delta\right) \operatorname{Re}_{\delta}^{1 / 2}$ & 0.010 & 0.008 & 0.001 & $\overline{S_{2}} /\left(\nu / \lambda_{\nu}^{2}\right)$ & 1.4 & 1.2 & 0.2 \\
\hline$C_{S 3}$ & $\overline{S_{3}} /\left(u_{c} / \delta\right) \operatorname{Re}_{\delta}^{1 / 2}$ & 0.088 & 0.088 & 0.041 & $\overline{S_{3}} /\left(\nu / \lambda_{\nu}^{2}\right)$ & 12.6 & 12.8 & 10.9 \\
\hline$C_{W}$ & $\overline{\omega_{i} \omega_{i}} /\left(u_{c} / \delta\right)^{2} \operatorname{Re}_{\delta}^{1 / 2}$ & 0.016 & 0.017 & 0.003 & $\overline{\omega_{i} \omega_{i}} /\left(\nu / \lambda_{\nu}^{2}\right)^{2}$ & 321 & 353 & 227 \\
\hline$C_{P}$ & $\overline{\omega_{i} S_{i j} \omega_{j}} /\left(u_{c} / \delta\right)^{3} \operatorname{Re}_{\delta}^{3 / 2}$ & $5.4\left(10^{-4}\right)$ & $5.0\left(10^{-4}\right)$ & $0.1\left(10^{-4}\right)$ & $\overline{\omega_{i} S_{i j} \omega_{j}} /\left(\nu / \lambda_{\nu}^{2}\right)^{3}$ & 1569 & 1538 & 91 \\
\hline$C_{S}$ & $2 \nu \overline{S_{i j} S_{i j}} /\left(u_{c}^{3} / \delta\right)$ & 0.061 & 0.062 & 0.015 & $2 \nu \overline{S_{i j} S_{i j}} /\left(\nu^{3} / \lambda_{\nu}^{4}\right)$ & 1404 & 1485 & 631 \\
\hline$C_{K}$ & $\lambda_{\nu} / \lambda_{K}$ & 6.1 & 6.2 & 5.9 & & $\cdots$ & $\cdots$ & $\cdots$ \\
\hline$\Lambda_{W}$ & $\left(\lambda_{\nu}\right)_{W} / \delta \operatorname{Re}_{\delta}^{-3 / 4}$ & 12.3 & $\ldots$ & $\ldots$ & & $\ldots$ & $\ldots$ & $\cdots$ \\
\hline$\Lambda_{\varepsilon}$ & $\left(\lambda_{\nu}\right)_{\varepsilon} / \delta \mathrm{Re}_{\delta}^{-3 / 4}$ & 14.7 & $\cdots$ & $\cdots$ & & $\cdots$ & $\cdots$ & $\cdots$ \\
\hline
\end{tabular}

mean strain rate on the vorticity. The widths of the innerscaled pdfs for the $S \neq 0$ case are substantially smaller than for the two $S=0$ cases, but the three vorticity components in Fig. 17 (bottom) show far greater similarity than did the corresponding shear components of the strain rate for the same case in Fig. 10 (bottom).

A further test of the scaling and isotropy in the vorticity fields is shown in Fig. 18, where the inner-scaled component values from the two $S=0$ cases at $\operatorname{Re}_{\delta}=6,000$ and 30,000 are directly compared. The result shows that, consistent with the fundamental scaling, similarity and isotropy that form the basis of classical theory, the scaled vorticity component distributions at these $\operatorname{Re}_{\delta}$ values are essentially identical in this turbulent shear flow.

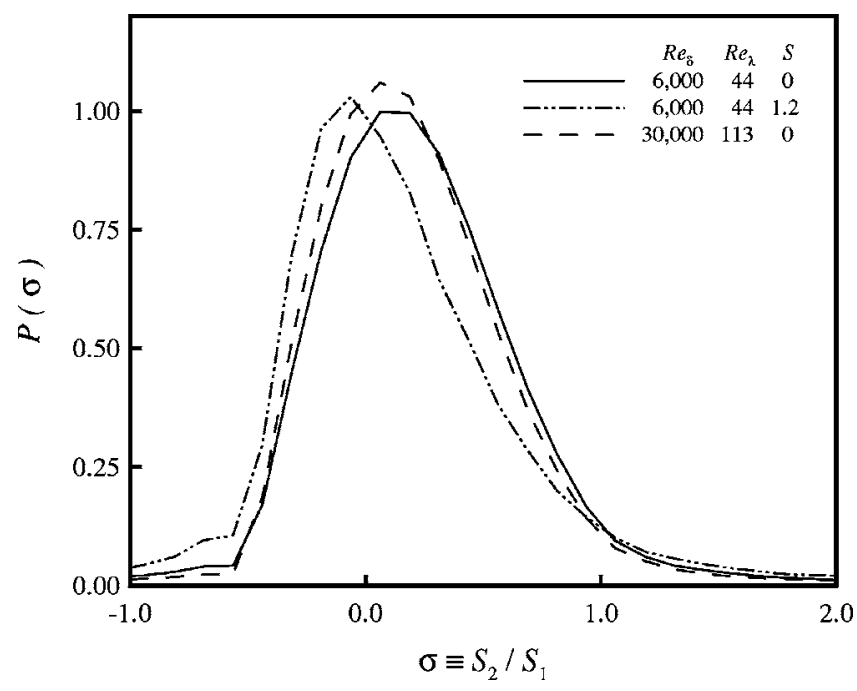

FIG. 13. Probability densities of strain rate structure parameter $\sigma$ $\equiv\left(S_{2} / S_{1}\right)$ for all three $\left(\operatorname{Re}_{\delta} ; S\right)$ cases, showing $\operatorname{Re}_{\delta}$ similarity for the two $S=0$ cases, and shift toward $\sigma<0$ for the $S=1.2$ case showing increase in strain rate eigenstates that lead to formation of line-like structures in the vorticity field.

\section{A. Vorticity alignment}

The origins of the anisotropy evident for the $S \neq 0$ case in Figs. 2-17 can be identified in the vorticity vector orientations relative to the original coordinate frame. The two spherical angles $\vartheta$ and $\varphi$ again define the orientation as in Sec. IV B, and in the absence of any alignment preference would follow the distributions in (3) and (4). Figures 19 and 20 give the measured distributions for each of the three cases, with the solid line giving the isotropic form. For the two $S=0$ cases, the $\varphi$ distributions in Fig. 19 generally follow the isotropic form, with only relatively small departures from isotropy that are attributed to the relatively weak mean shear across the field-of-view on either side of the flow centerline. However the $S \neq 0$ case shows a very strong departure from the isotropic form, with a large peak near $\varphi$ $\approx \pi / 2$, that corresponds to a strong preference for the vorticity vector to point along the most extensional principal strain axis of the mean strain rate field. The corresponding $\vartheta$ distributions in Fig. 20 show no significant alignment preference, consistent with the fact that the mean strain field imposes no preference for any $\vartheta$ orientation.

Figure 21 gives the orientation of the vorticity vector with respect to the strain rate eigenvectors for each of the three cases. Shown are distributions of the orientation cosines $\hat{e}_{i} \cdot \hat{e}_{\omega}$ between the strain rate eigenvector $\hat{e}_{i}$ and the vorticity unit vector $\hat{e}_{\omega}$. Comparing the two $S=0$ cases at $\operatorname{Re}_{\delta}=6,000$ and 30,000 shows that the vorticity orientation with respect to the local strain rate tensor is essentially the same in both cases. The vorticity is most strongly aligned with the eigenvector $\hat{e}_{2}$ corresponding to the intermediate principal strain rate $S_{2}$, and shows a preference to align perpendicular to the eigenvector $\hat{e}_{3}$ corresponding to the most compressional principal strain rate $S_{3}$. There is virtually no correlation between the vorticity and the eigenvector $\hat{e}_{1}$ that corresponds to the most extensional principal strain rate $S_{1}$. The detailed forms of these two sets of $S=0$ distributions 

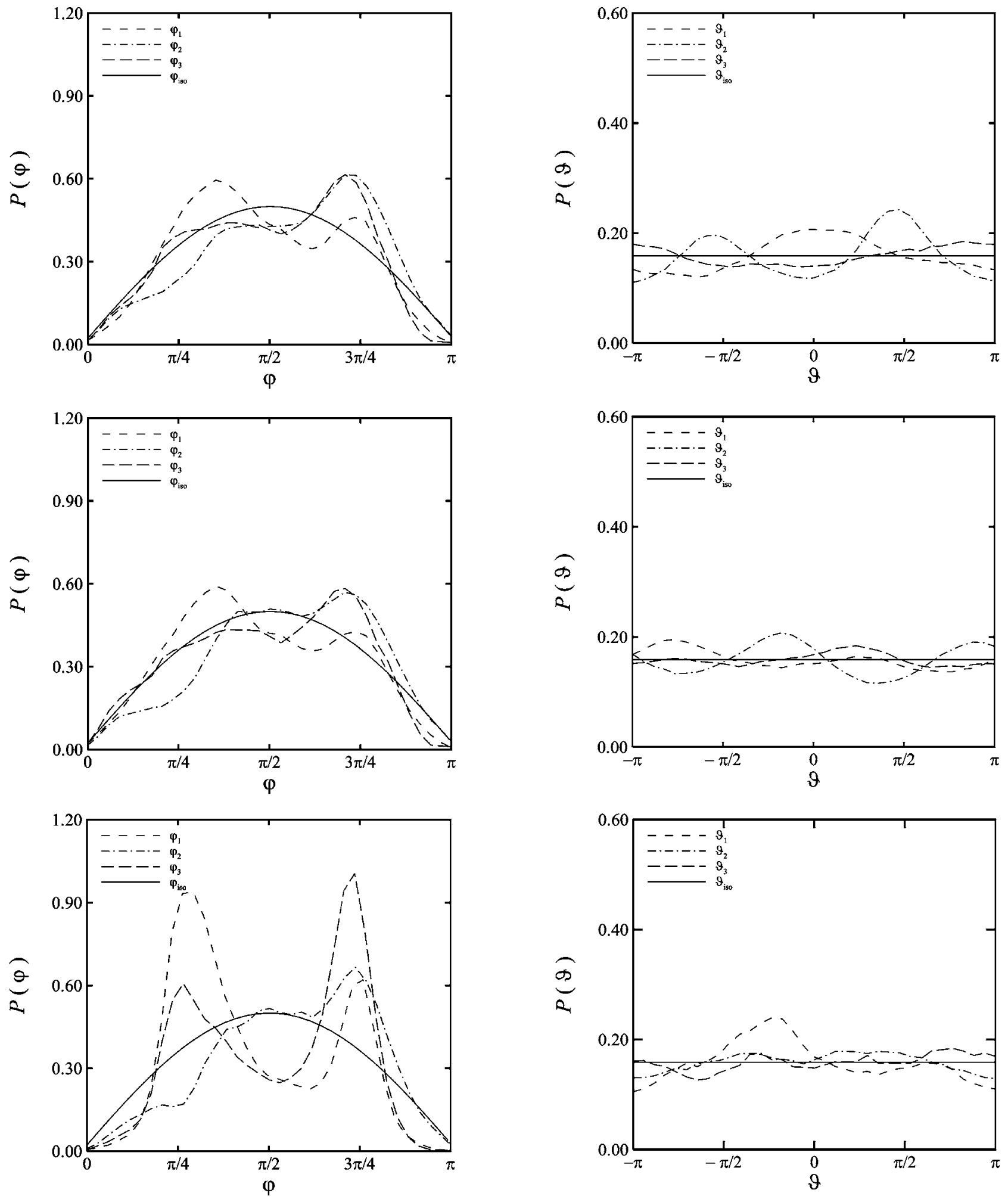

FIG. 14. Probability densities of the orientation angle $\varphi$ for each of the three principal strain axes $\hat{e}_{i}$ for all three $\left(\operatorname{Re}_{\delta} ; S\right)$ cases: $(6,000 ; 0)$ (top), $(30,000$; $0)$ (middle), and $(6,000 ; 1.2)$ (bottom) with the solid line giving the isotropic result.

agree with numerical simulations of homogeneous isotropic turbulence $^{28}$ and with previous measurements. ${ }^{24,29-31}$

The alignment preference in Figs. 21 (top, middle) is consistent with simple kinematic considerations in the geometric statistics of turbulence. ${ }^{32}$ A concentrated vortical
FIG. 15. Probability densities of the orientation angle $\vartheta$ for each of the three principal strain axes $\hat{e}_{i}$ for all three $\left(\operatorname{Re}_{\delta} ; S\right)$ cases: $(6,000 ; 0)$ (top), $(30,000$; $0)$ (middle), and $(6,000 ; 1.2)$ (bottom) with the solid line giving the isotropic result.

structure has a preferred orientation relative to the eigenvectors $\hat{e}_{i}^{B}$ of the local background strain field $S_{i j}^{B}$ created by all other vortical structures in the flow. The vortex stretching term $\omega_{i} S_{i j}^{B}$ will cause the structure to rotate away from the most compressional principal strain axis $\hat{e}_{3}^{B}$ of the back- 

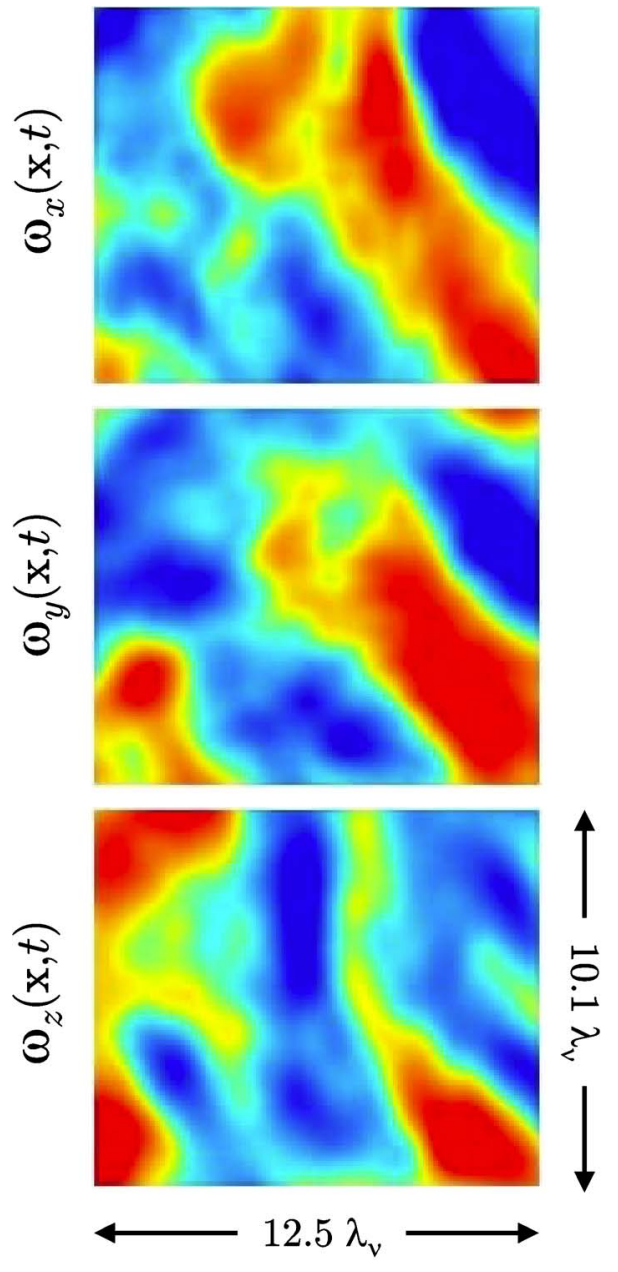

$-30.0 \frac{\omega_{i}}{\left(v / \lambda_{v}{ }^{2}\right)} \quad 30.0$

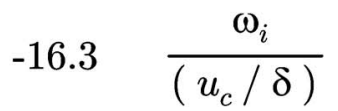

16.3

FIG. 16. (Color online) Typical three-component vorticity field $\omega_{i}(\mathbf{x}, t)$ at $\left(\operatorname{Re}_{\delta} ; S\right)=(6,000 ; 0)$. The field of view is shown normalized by the local inner length scale $\lambda_{v}$. The color scale shows quantitative values normalized on local inner $\left(\nu, \lambda_{\nu}\right)$ and outer $\left(u_{c}, \delta\right)$ scales.

ground strain field and toward the most extensional principal axis $\hat{e}_{1}^{B}$. This might suggest a different alignment than that seen in Fig. 21, however these are distributions for the orientation relative to the eigenvectors $\hat{e}_{i}$ of the total strain rate field, consisting of the sum of the background strain and the local strain induced by the structure. The latter is twodimensional, and has eigenvectors in the plane normal to the structure. Thus even though the vorticity rotates toward alignment with $\hat{e}_{1}^{B}$, if the circulation of the vortical structure is large enough relative to the background strain rate $S^{B}$ $=\left(S_{i j}^{B} S_{i j}^{B}\right)^{1 / 2}$, then the most extensional principal axis of the combined strain field in the vicinity of the structure will be in the plane perpendicular to $\hat{e}_{1}^{B}$, which corresponds to the orientations seen in Fig. 21.
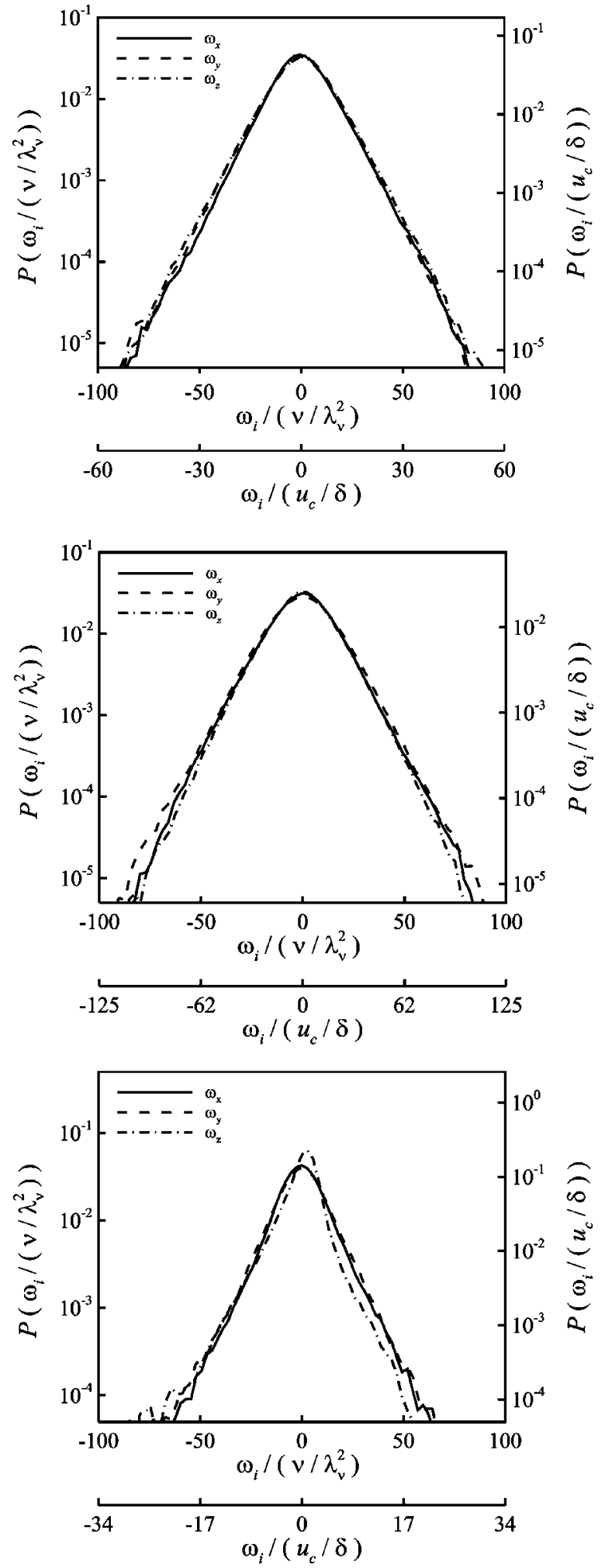

FIG. 17. Probability densities of vorticity vector components $\omega_{i}$ at $\left(\operatorname{Re}_{\delta} ; S\right)=(6,000 ; 0)$ (top), $(30,000 ; 0)$ (middle), and $(6,000 ; 1.2)$ (bottom) shown scaled on local inner $\left(\nu, \lambda_{\nu}\right)$ and outer $\left(u_{c}, \delta\right)$ variables.

The eigenvectors $\hat{e}_{i}^{B}$ vary with time, and the vorticity reorients itself on the time scale $1 / S^{B}$. Thus if the time scale $\tau^{B}$ on which $S_{i j}^{B}$ varies satisfies $\tau^{B} \gg 1 / S^{B}$ then the reorientation dynamics due to vortex stretching cannot keep up with the changes in $S_{i j}^{B}$, and there will be no preferred alignment 


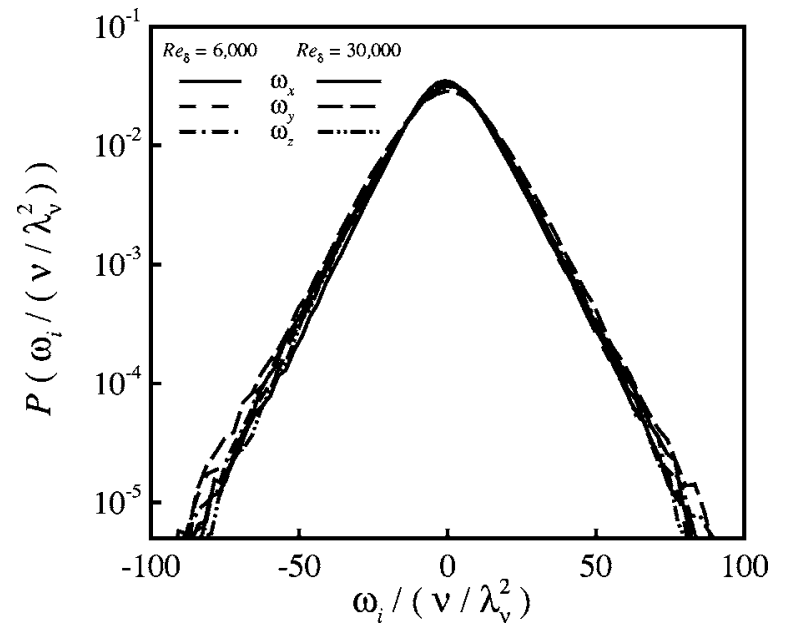

FIG. 18. Probability densities of all three vorticity vector components $\omega_{i}$ for both $S=0$ cases at $\operatorname{Re}_{\delta}=6,000$ and 30,000, shown scaled on inner variables $\left(\nu, \lambda_{\nu}\right)$ to test $\operatorname{Re}_{\delta}$ similarity and isotropy.

of the vorticity with the eigenvectors $\hat{e}_{i}$ of the combined strain field. On the other hand, if $\tau^{B} \ll 1 / S^{B}$ then the preferred alignment noted above will be maintained. The fact that a strong preferred alignment is seen for the two $S=0$ cases in Fig. 21 indicates that $\tau^{B} \ll 1 / S^{B}$ occurs far more often, with the consequence that the vorticity maintains a significant but imperfect alignment with the local instantaneous eigenvectors of the background strain field.

For the $S \neq 0$ case in Fig. 21, the relative alignments are significantly different than in the two $S=0$ cases. This can be understood from the stationary mean strain field $S_{i j}^{M}$ imposed by the mean shear, which acts in addition to the time-varying background strain field $S_{i j}^{B}$ to reorient the vortical structures. When $S_{i j}^{M} \ll S_{i j}^{B}$ the vorticity will tend toward alignment with $\hat{e}_{1}^{B}$, while if $S_{i j}^{M} \gg S_{i j}^{B}$ the vorticity would align with $\hat{e}_{1}^{M}$. As a result, for the $S \neq 0$ case in Fig. 21, the vorticity alignment with respect to the eigenvectors of the total strain field shows roughly similar trends as in the two $S=0$ cases, but with much weaker preference to maintain this alignment.

\section{B. Enstrophy and enstrophy production rate}

Figure 22 gives typical examples of the instantaneous enstrophy fields $\omega_{i} \omega_{i}(\mathbf{x}, t)$ in each of the three cases, showing the highly intermittent structures into which such secondorder gradient quantities are concentrated by the underlying strain-diffusion competition noted in Sec. I. Owing to the fixed field of view of the measurements and the $\delta$ values in Table I, the relative size of the $\mathrm{Re}_{\delta}=30,000$ planes is nearly twice that of the $\operatorname{Re}_{\delta}=6,000$ planes.

Probability densities of the scaled enstrophy values are given in Fig. 23. For the two $S=0$ cases it can be seen that the enstrophy values scaled on inner variables with $\left(\nu / \lambda_{\nu}^{2}\right)^{2}$ are essentially identical at $\operatorname{Re}_{\delta}=6,000$ and 30,000. When scaled on outer variables with $\left(u_{c} / \delta\right)^{2}$ the values at $\operatorname{Re}_{\delta}$ $=6,000$ and 30,000 differ by a factor of 5, in agreement with the result from $\lambda_{\nu} / \delta \sim \operatorname{Re}_{\delta}^{-3 / 4}$ giving $\left(\nu / \lambda_{\nu}^{2}\right)^{2} \sim\left(u_{c} / \delta\right)^{2} \operatorname{Re}_{\delta}$. It is also apparent that the scaled enstrophy distribution from
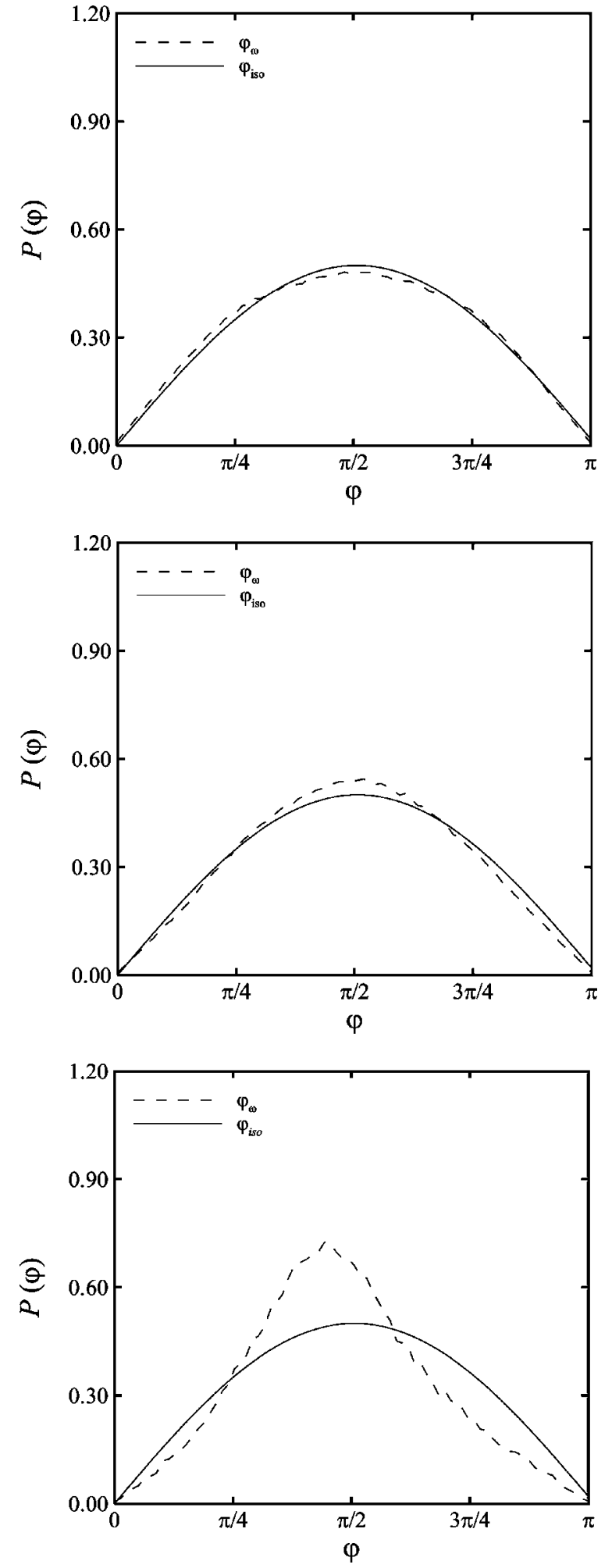

FIG. 19. Probability densities of the orientation angle $\varphi$ for the vorticity vector field $\boldsymbol{\omega}(\mathbf{x}, t)$ for $\left(\operatorname{Re}_{\delta} ; S\right)=(6,000 ; 0)$ (top), $(30,000 ; 0)$ (middle), and $(6,000,1.2)$ (bottom), with solid line giving isotropic distribution.

the $S \neq 0$ case is significantly different than for the two $S$ $=0$ cases. Scaling coefficients for the mean enstrophy values for all three cases are given in Table IX.

The dashed lines in Fig. 23 give log normal fits with the same first two moments as the measured distributions. In all three cases, the measured enstrophy values are seen to be 

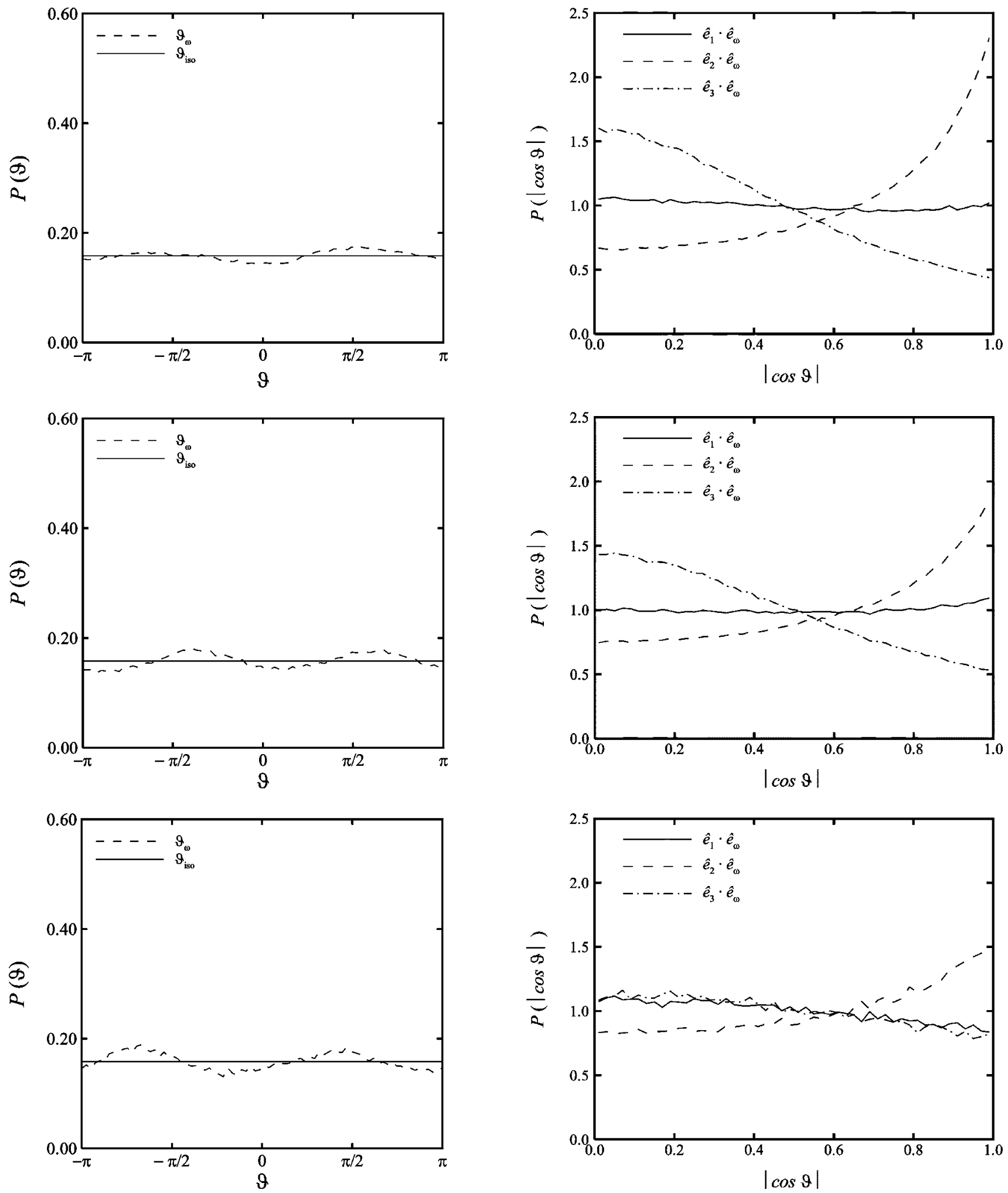

FIG. 20. Probability densities of the orientation angle $\vartheta$ for the vorticity vector field $\boldsymbol{\omega}(\mathbf{x}, t)$ for $\left(\operatorname{Re}_{\delta} ; S\right)=(6,000 ; 0)$ (top), $(30,000 ; 0)$ (middle), and $(6,000 ; 1.2)$ (bottom), with solid line giving isotropic distribution.

nearly log normally distributed, though consistent small departures from strict log normality are evident. The origins of these may be related to the nonergodic properties of random multiplicative cascades, which are shown in Sec. VIII to describe the spatial scale similarity in these enstrophy fields. In

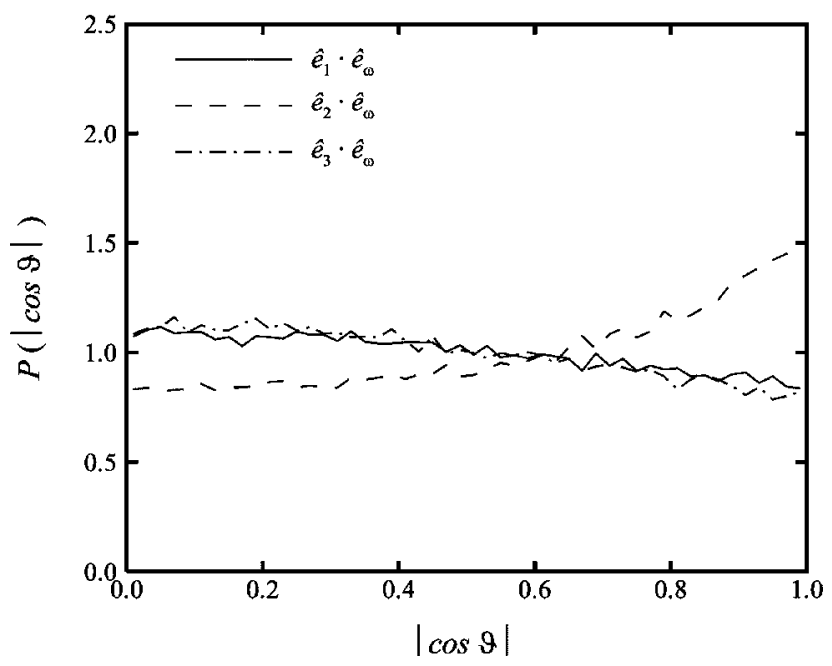

FIG. 21. Probability densities of local alignment cosines between vorticity vector $\boldsymbol{\omega}(\mathbf{x}, t)$ and each of the three principal strain axes $\hat{e}_{i}$ of the strain rate field $\varepsilon_{i j}(\mathbf{x}, t)$, for $\left(\operatorname{Re}_{\delta} ; S\right)=(6,000 ; 0)$ (top), $(30,000 ; 0)$ (middle), and $(6,000$; 1.2) (bottom).

such multiplicative cascades, distributions resulting from ensembles of independent samples will be log normal, whereas distributions from spatial samples reflect the spatial correlations inherent in such cascades. The distributions in Fig. 23 

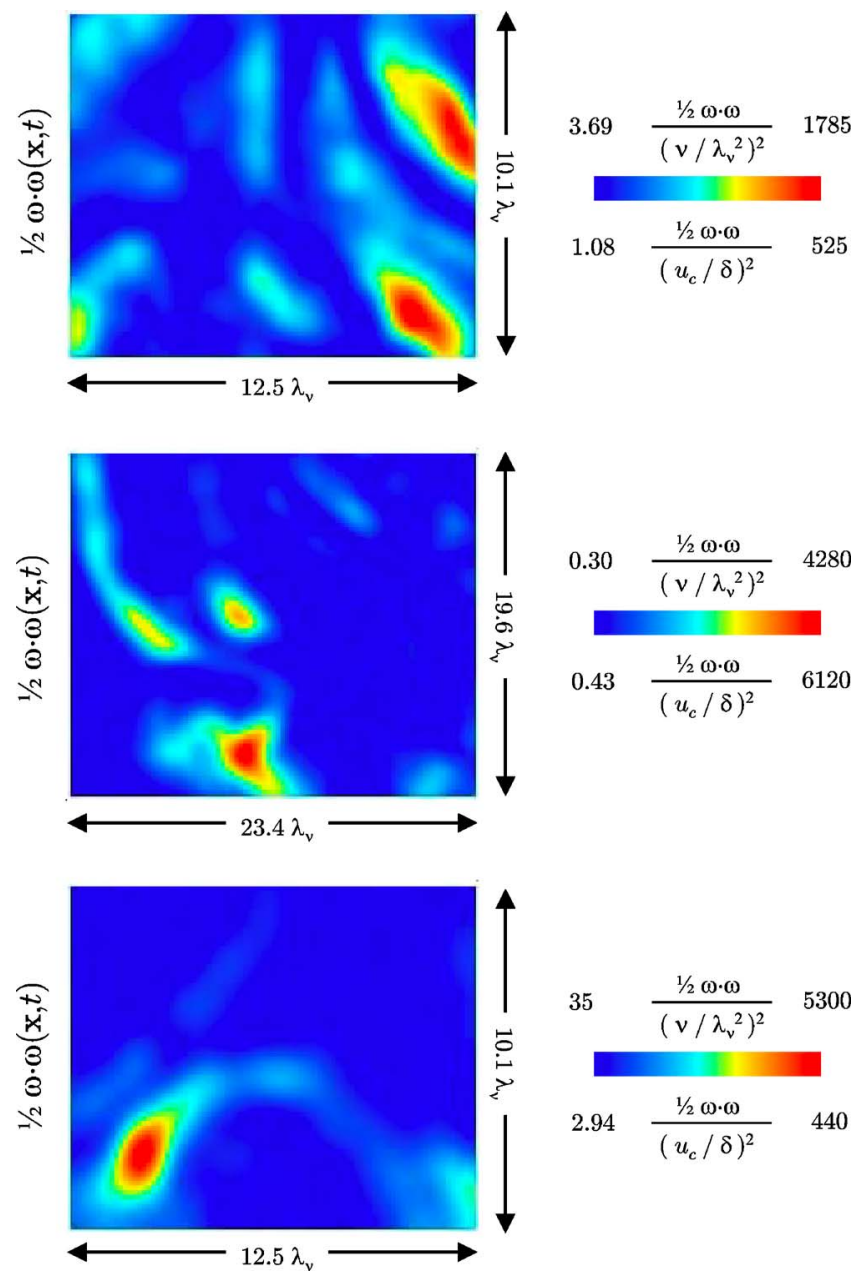

FIG. 22. (Color online) Typical measured enstrophy fields $\omega_{i} \omega_{i}(\mathbf{x}, t)$ at $\left(\operatorname{Re}_{\delta} ; S\right)=(6,000 ; 0)$ (top), $(30,000 ; 0)$ (middle), and $(6,000 ; 1.2)$ (bottom).

for the two $S=0$ cases result from a mixture of spatial and ensemble statistics, involving roughly 1000 velocity gradient values in each spatial data plane and roughly 1000 independent planes in each ensemble, and thus departures from strict $\log$ normality may be expected.

The local enstrophy production rate $\omega_{i} S_{i j} \omega_{j}(\mathbf{x}, t)$ is a third-order gradient quantity and thus based on classical theory would show a rapid increase in intermittency with increasing outer-scale Reynolds number, since the inner scaling $\left(\nu / \lambda_{\nu}^{2}\right)^{3}$ corresponds to the outer scaling $\left(u_{c} / \delta\right)^{3} \operatorname{Re}_{\delta}^{3 / 2}$. Figure 24 gives an example of the measured enstrophy production rate field for each of the three cases. From the outer scaling above, the production rate values scaled on $\left(u_{c} / \delta\right)^{3}$ should be 11.1 times larger at $\operatorname{Re}_{\delta}=30,000$ than at $\operatorname{Re}_{\delta}$ $=6,000$ for $S=0$. This appears consistent with the scales in Fig. 24, and can be verified in the measured distributions in Fig. 25. The scaling coefficient for the mean enstrophy production rate can be seen in Table IX to be essentially the same in the two $S=0$ cases, but is far smaller for the $S \neq 0$ case. This is consistent with the positive skewness for the $S=0$ cases seen in the semilogarithmic axes on the right in Fig. 25. In contrast, the $S=0$ cases show nearly symmetric distributions, consistent with the result in Fig. 21 showing far poorer alignment of the vorticity with the eigenvectors of the strain rate tensor.

\section{KINETIC ENERGY DISSIPATION RATE}

Figure 26 shows the measured kinetic energy dissipation rate field $2 \nu \varepsilon_{i j} \varepsilon_{i j}(\mathbf{x}, t)$ for all three cases, in the same plane as for the corresponding enstrophy and production rate fields in Figs. 22 and 24. Since the two $\operatorname{Re}_{\delta}=6,000$ cases are fully resolved these are true dissipation rate fields, while the $\mathrm{Re}_{\delta}$ $=30,000$ case is nearly fully resolved. This can be verified by the corresponding scalings on inner and outer variables. Note that the appropriate inner-variable scaling $\left(\nu^{3} / \lambda_{v}^{4}\right)$ for the dissipation is equivalent to the outer-variable scaling $\left(u_{c}^{3} / \delta\right)$, and is thus independent of $\operatorname{Re}_{\delta}$. This can be verified in the measured distributions in Fig. 27, where the measured dissipation rates for the two $S=0$ cases when normalized on both the inner- and outer-variable scalings are indeed virtually identical at $\operatorname{Re}_{\delta}=6,000$ and 30,000. Table IX gives the scaling coefficients for the mean dissipation rate for all three cases.

The dashed lines in Fig. 27 give log normal fits to the measured distributions. In all three cases, the measured dissipation rate values can be seen to be roughly log normally distributed, with the largest departures seen for the $S \neq 0$ case, though consistent departures from strict log normality are evident in the semilogarithmic forms. As noted for the enstrophy distributions in Fig. 25, this may be attributable to the nonergodic properties of random multiplicative cascades, which are shown in Sec. VIII to also apply to the spatial scale similarity in these dissipation rate fields. As a consequence, the smaller ensemble of independent data planes in the $S \neq 0$ case leads to probability distributions dominated by spatial statistics, in which spatial correlations can lead to departures from log normality. The two $S=0$ cases involve a much larger ensemble of independent planes, with the resulting distributions are thus more strongly influenced by ensemble statistics that produce log normal distributions.

\section{INNER LENGTH SCALES}

Values for the viscous length scale $\lambda_{\nu}$ used for innervariable normalizations throughout these results were obtained from the relation $\left(\lambda_{\nu} / \delta\right)=\Lambda \operatorname{Re}_{\delta}^{-3 / 4}$, with the mean scaling constant $\langle\Lambda\rangle \approx 11.2$ from Refs. 5-9. However, the $\Lambda$ values from these earlier studies were obtained indirectly from length scales in the scalar dissipation rate field $D \nabla \zeta \cdot \nabla \zeta(\mathbf{x}, t)$ via measurements of dynamically passive conserved scalar fields $\zeta(\mathbf{x}, t)$. The corresponding gradient length scale $\lambda_{D}$ results from the competing effects of the local strain rate $S_{i j}$ and the scalar diffusivity $D$. A similar competition between $S_{i j}$ and viscous diffusion leads to the viscous length scale $\lambda_{\nu}$ in the velocity gradient fields, as noted in Sec. I. If there were no other differences in these two strain-diffusion processes, then the ratio of the scalar and viscous diffusion length scales would be $\left(\lambda_{D} / \lambda_{\nu}\right)=\mathrm{Sc}^{-1 / 2}$, where $\mathrm{Sc} \equiv(\nu / D)$. All measured values of $\Lambda$ to date have been based on this assumption. 

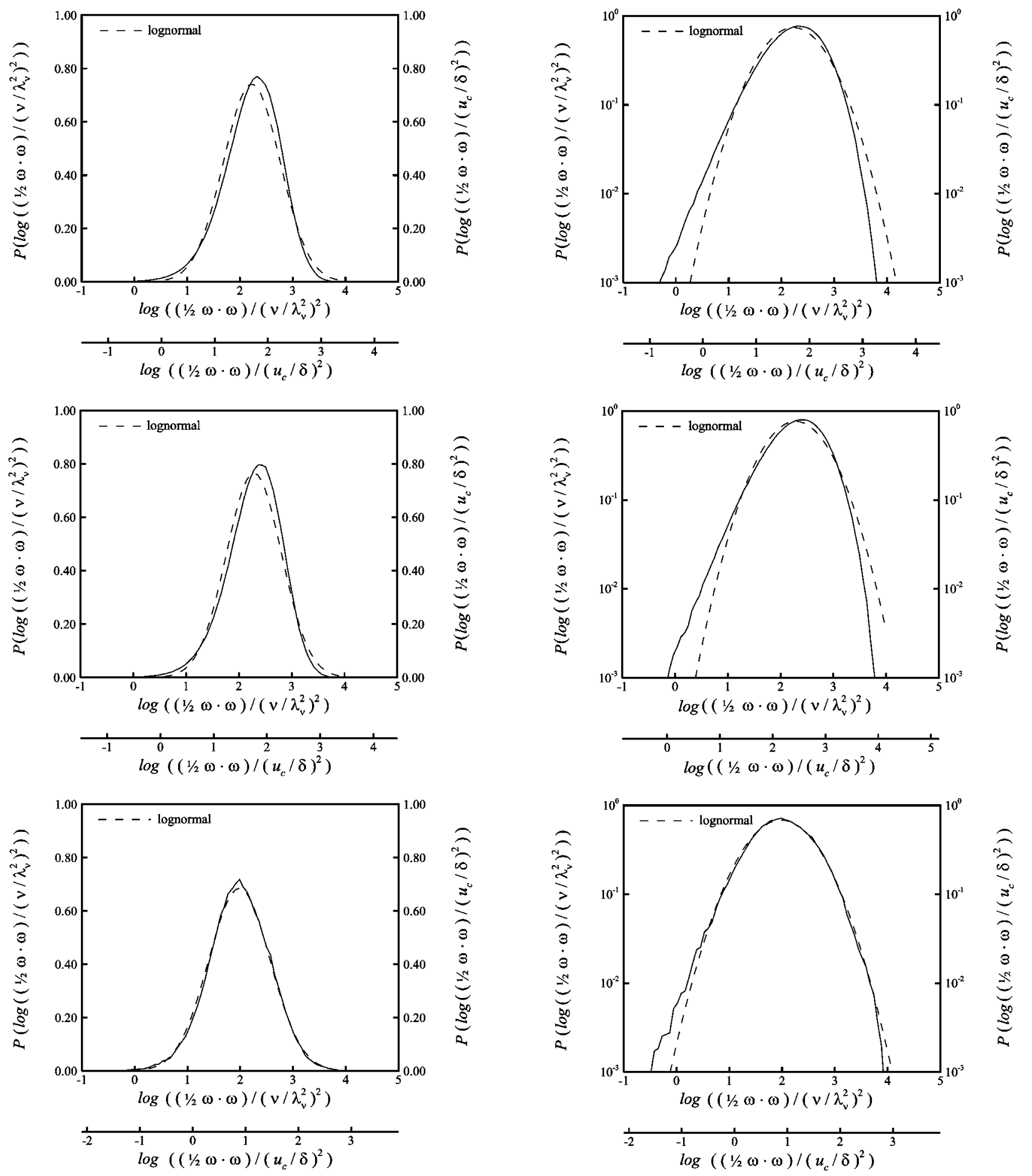

FIG. 23. Probability densities of the enstrophy $\omega_{i} \omega_{i}$, scaled on local inner $\left(\nu, \lambda_{\nu}\right)$ and outer $\left(u_{c}, \delta\right)$ variables for $\left(\operatorname{Re}_{\delta} ; S\right)=(6,000 ; 0)($ top $),(30,000 ; 0)(\operatorname{middle})$, and $(6,000 ; 1.2)$ (bottom), with dashed lines giving corresponding log normal fits.

However there are differences in the two strain-diffusion processes beyond the simple difference in $\nu$ and $D$. Different geometries are associated with the diffusive-scale structures in the velocity gradient field than in the scalar gradient field, which must be taken into account when inferring $\lambda_{\nu}$ values from $\lambda_{D}$. The conserved scalar dynamics lead to scalar gradients that form solely into sheet-like diffusive-scale structures, while the vorticity dynamics allows both line-like and sheet-like diffusive-scale structures. ${ }^{5,6}$ Consequently, earlier indirect measurements of $\Lambda$ provided accurate results for the scalar gradient length scale $\left(\lambda_{D} / \delta\right)=\Lambda_{D} \operatorname{Re}_{\delta}^{-3 / 4} \mathrm{Sc}^{-1 / 2}$, but the different inner-scale geometries prevent direct inference of corresponding $\Lambda_{\nu}$ values from $\Lambda_{D}$ measurements.

The present velocity gradient results allow measurement of $\lambda_{\nu}$ in a turbulent shear flow to determine the corresponding inner scaling constant $\Lambda_{\nu}$. One-dimensional intersections 

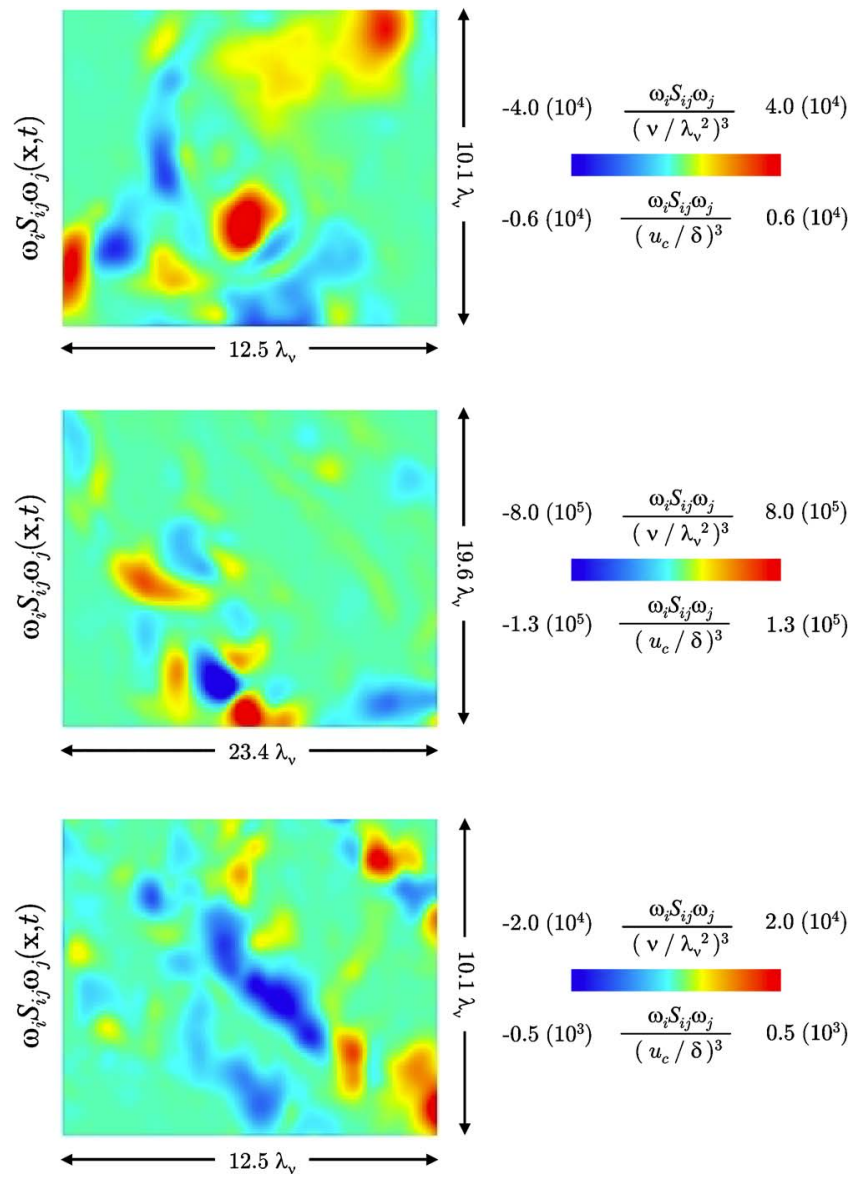

FIG. 24. (Color online) Typical measured enstrophy production rate fields $\omega_{i} S_{i j} \omega_{j}(\mathbf{x}, t)$ scaled on local inner $\left(\nu, \lambda_{\nu}\right)$ and outer $\left(u_{c}, \delta\right)$ variables for $\left(\operatorname{Re}_{\delta} ; S\right)=(6,000 ; 0)$ (top), $(30,000 ; 0)$ (middle), and $(6,000 ; 1.2)$ (bottom).

corresponding to every row and column in the enstrophy and dissipation rate fields were analyzed for the $\mathrm{Re}_{\delta}=6,000$ case with $S=0$. In each intersection all local maxima were identified, and the distance $\left(\lambda_{\nu}\right)_{50 \%}$ on either side required for the field to decrease to one-half of the local maximum value was found. Instances in which a local minimum was reached on either side before the field had decreased to the local halfmaximum value were rejected, since these correspond to merging of adjacent inner-scale structures.

Figure 28 shows probability densities for the resulting $\left(\lambda_{\nu}\right)_{50 \%}$ values in the enstrophy and dissipation rate fields. Small differences between these distributions are expected due to the different types of fields considered in each panel; Ref. 33 has also proposed different inner length scales for various fields. The resulting distributions of one-dimensional gradient length scales $\left(\lambda_{\nu}\right)_{1 \mathrm{D}}$ can be deconvolved to obtain the corresponding distributions of true three-dimensional gradient length scales $\left(\lambda_{v}\right)_{3 \mathrm{D}}$ based on the geometry and isotropy of the inner-scale structures. ${ }^{34,35}$ The assumed isotropy is justified for the $S=0$ case. Regarding the inner-scale geometry, this involves the fraction of intersections through sheet-like $(\sigma \rightarrow 1)$, line-like $(\sigma \rightarrow-1 / 2)$, and other structures, though the effect of these is relatively small. The solid lines in Fig. 28 show the resulting distributions of $\left(\lambda_{\nu}\right)_{50 \%}$, with the deconvolution based on sheet-like structures. The distri- butions obtained for the enstrophy and dissipation rate fields are very similar, with the largest $\lambda_{\nu}$ values exceeding the smallest by only about a factor of 3 .

From the resulting mean values $\left\langle\left(\lambda_{v}\right)_{3 \mathrm{D}}\right\rangle$ in Fig. 28, rescaled to the standard $\left(\lambda_{\nu}\right)_{20 \%}$ definition noted in Sec. I, corresponding values for the scaling constant $\left\langle\Lambda_{\nu}\right\rangle$ can be obtained from $\left\langle\left(\lambda_{\nu}\right)_{3 \mathrm{D}}\right\rangle / \delta=\left\langle\Lambda_{\nu}\right\rangle \operatorname{Re}_{\delta}^{-3 / 4}$. This gives $\left\langle\Lambda_{\nu}\right\rangle \approx 12.3$ in the enstrophy field and $\left\langle\Lambda_{\nu}\right\rangle \approx 14.7$ in the dissipation rate field, as shown in Table IX. The differences result from the differing diffusive scale structures in these fields; in principle a different $\left\langle\Lambda_{\nu}\right\rangle$ value should apply to each field. The present results for $\left\langle\Lambda_{\nu}\right\rangle$ are slightly larger than the previous indirect estimates based on $\lambda_{D}$ measurements, ${ }^{5-9}$ and represent the first direct measurements of $\Lambda_{\nu}$ in a turbulent flow.

The present measurements also provide the true mean kinetic energy dissipation rate $\varepsilon$ in the two fully resolved cases at $\operatorname{Re}_{\delta}=6,000$, and provide a nearly resolved value for $\varepsilon$ in the $\operatorname{Re}_{\delta}=30,000$ case. These allow the resulting Kolmogorov scale $\lambda_{K} \equiv\left(\nu^{3} / \varepsilon\right)^{1 / 4}$ to be obtained without any additional assumptions. The measured mean dissipation rates $\varepsilon$ for each case in Table I give the ratio $\lambda_{\nu} / \lambda_{K} \approx 6.1$ as indicated in Table IX, verifying that the Kolmogorov scale is about a factor of 6 smaller than the physical size of the inner-scale structures.

\section{SCALE SIMILARITY}

Previous studies have examined intermittency corrections to the classical theory based on multifractal scale similarity in various surrogates for the true enstrophy and kinetic energy dissipation rate fields. ${ }^{36-38}$ The present direct measurements of these fields flow allow a rigorous test for such scale similarity in a turbulent shear.

\section{A. Multifractal scaling}

Multifractal fields result from the repeated application of a scale-invariant multiplicative process to an initial field. In turbulent flows, such a multiplicative process is produced by the continual stretching and folding action of the timevarying strain rate and vorticity fields, with the required scale invariance being satisfied for scales sufficiently smaller than the local outer scale $\delta$. Such scale-invariant multiplicative processes can be represented by deterministic or stochastic multiplicative cascades in which a fixed set of multipliers $M$ maps the field from one iteration to the next as the cascade proceeds. In one-dimensional stochastic cascades, at each successive stage in the process the total amount $m$ of the field quantity contained in any given cell is distributed over two cells, each half the size of the previous stage, with the multiplier $0 \leqslant M \leqslant 1$ that determines the division between the two cells obtained randomly from a scale-invariant distribution $P(M)$. After a sufficient number of such repetitions, the resulting field $m(x)$ becomes highly intermittent and displays multifractal scaling properties.

The same process can be applied "in reverse" to test for multifractal scale similarity in a given field. Onedimensional extracts from the field are repeatedly integrated over increasingly larger cell sizes $r$, with the cell size doubling between successive stages in the process. At each stage 

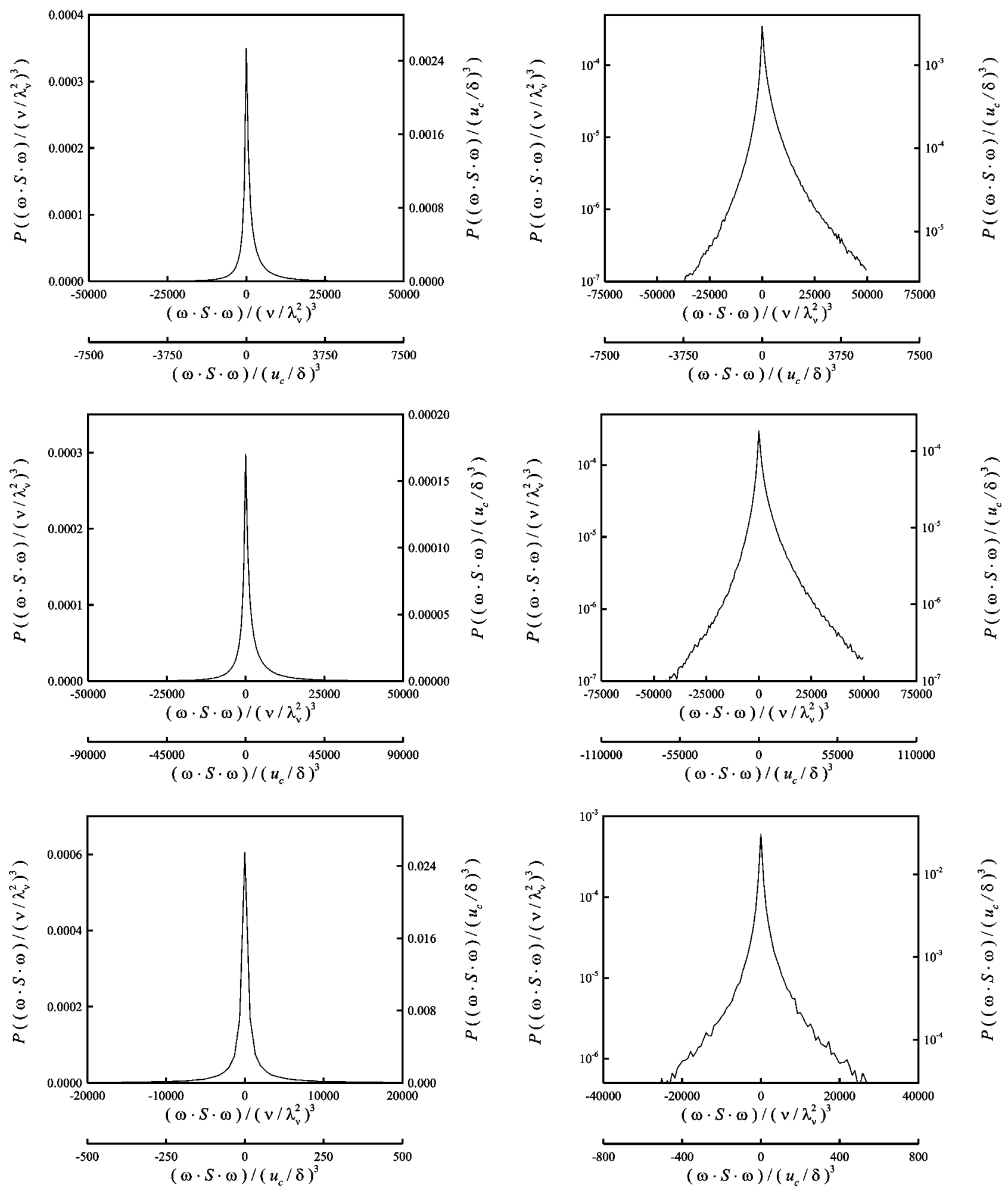

FIG. 25. Probability densities of enstrophy production rates $\omega_{i} S_{i j} \omega_{j}$, scaled on inner $\left(\nu, \lambda_{\nu}\right)$ and outer $\left(u_{c}, \delta\right)$ variables in linear (left) and semilog (right) axes for $\left(\operatorname{Re}_{\delta} ; S\right)=(6,000 ; 0)$ (top), $(30,000 ; 0)$ (middle), and $(6,000 ; 1.2)$ (bottom).

$i$, in each cell $j$ the ratio of the integrated value $\left(m_{j}\right)_{i}$ to the integrated value $\left(m_{j}\right)_{(i-1)}$ is the local multiplier value 0 $\leqslant\left(M_{r}\right)_{j} \leqslant 1$ at the scale $r$. The ensemble of multiplier values from the $j=1 \ldots 2^{i}$ cells at the scale $r=2^{-i}$ determines the multiplier distribution $P\left(M_{r}\right)$ at that scale. If the field is multifractal then the resulting $P\left(M_{r}\right)$ will be the same at all scales $r$ where the underlying multifractal scale invariance applies. Generating the set of multiplier distributions $P\left(M_{r}\right)$ from such one-dimensional intersections through the field of interest provides a direct way to test for multifractal scale similarity in the field. If the field is shown to be multifractal through scale invariance in its distributions $P\left(M_{r}\right)$, then the resulting scale-invariant multiplier distribution $P(M)$ determines all the scaling properties of the field, including its moments $M_{q}$ of all orders $q$, and the associated scaling exponents $\tau(q)$. 

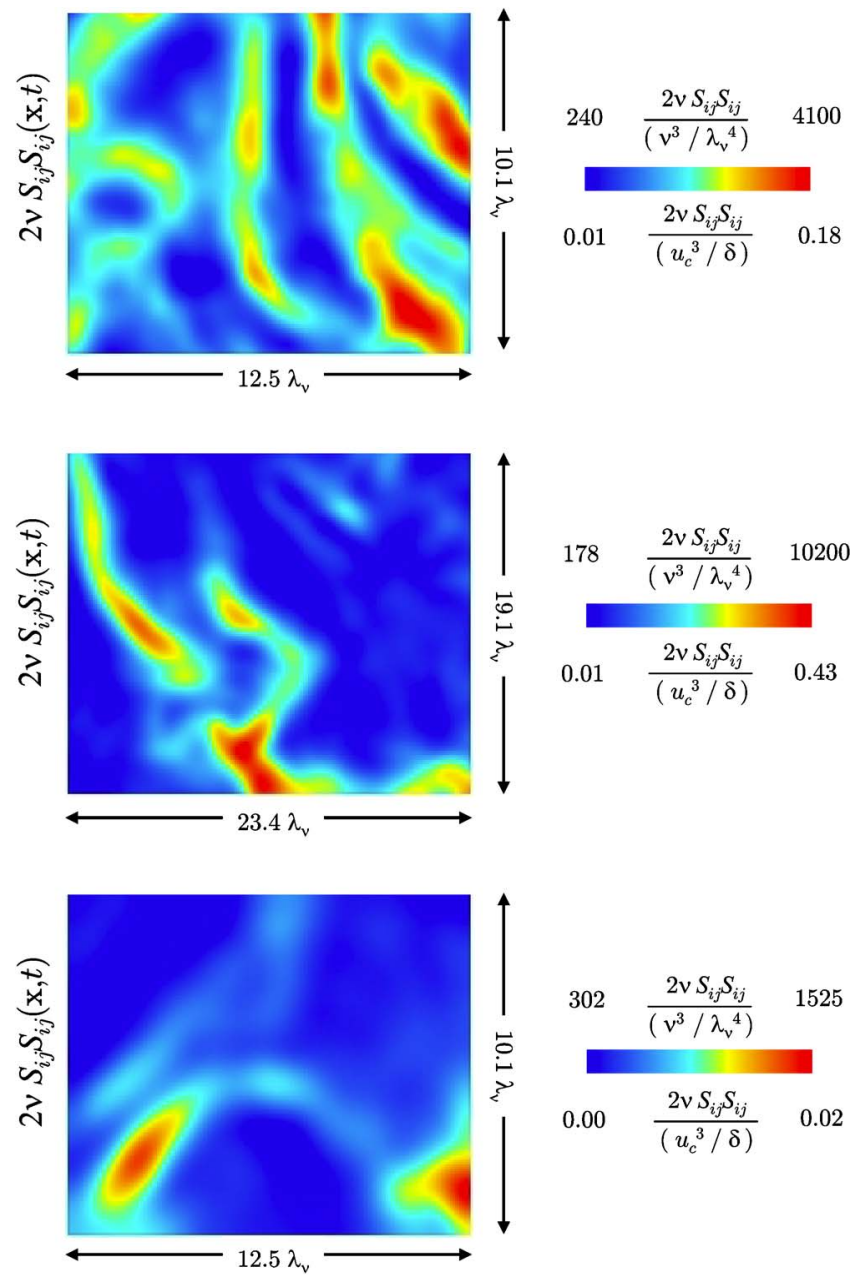

FIG. 26. (Color online) Typical measured kinetic energy dissipation rate fields $2 \nu S_{i j} S_{i j}(\mathbf{x}, t)$ at $\left(\operatorname{Re}_{\delta} ; S\right)=(6,000 ; 0)$ (top), (30,000;0) (middle), and $(6,000 ; 1.2)$ (bottom).

\section{B. Results for enstrophy and dissipation fields}

Figure 29 gives the resulting multiplier distributions $P\left(M_{r}\right)$ at each scale $\left(r / \lambda_{v}\right)$ from enstrophy fields of the type in Fig. 22 for the $\operatorname{Re}_{\delta}=6,000$ and 30,000 cases with $S=0$. Corresponding results from dissipation rate fields of the type in Fig. 26 for the same two cases are given in Fig. 30. Note first that as $r / \lambda_{v} \rightarrow 0$ the multiplier distributions $P\left(M_{r}\right)$ must converge to the trivial result $M \rightarrow 1 / 2$ since the fields become increasingly uniform for $r \ll \lambda_{\nu}$. Evidence of the approach to this trivial limit can be seen in the $P\left(M_{r}\right)$ distributions in both Figs. 29 and 30 for $\left(r / \lambda_{v}\right) \leqslant 1.3$. Moreover, the similarities in the form of $P\left(M_{r}\right)$ at the same $\left(r / \lambda_{v}\right)$ in these figures at $\operatorname{Re}_{\delta}=6,000$ and 30,000, for which the relative measurement resolution differs by nearly a factor of 2 (see Table I), strongly indicates that the departures from the scale invariance seen at larger scales is due to the viscous cutoff, and not due to measurement resolution. This further verifies that $\lambda_{\nu}$ is the appropriate physical length scale at which the viscous cutoff occurs in the scaling of turbulent flows.

For $\left(r / \lambda_{\nu}\right) \geqslant 2.5$, the results in Figs. 29 and 30 show clear evidence of multifractal scale similarity in the enstrophy and dissipation rate fields. At $\operatorname{Re}_{\delta}=6,000$ in the top panel of each figure, the multiplier distributions at the three largest scales shown, corresponding to $\left(r / \lambda_{\nu}\right)=2.5,5.1$ and 10.3, are clearly scale invariant. Moreover for both the enstrophy and the dissipation fields, the forms of the corresponding $P\left(M_{r}\right)$ over this scale range are essentially identical to the results found for $\operatorname{Re}_{\delta}=30,000$ in the lower panel of each figure at the four largest scales $\left(r / \lambda_{v}\right)=2.5,5.0,10.0$ and 20.0. The scale invariance in these $P\left(M_{r}\right)$ distributions over the same scale range $\left(r / \lambda_{\nu}\right)$ from independent measurements at different $\operatorname{Re}_{\delta}$ values confirms that the enstrophy and dissipation fields are multifractal, and gives the respective scaleinvariant forms of $P(M)$ that determine their scaling properties. $^{45}$

These present results also show the scale-invariant form of $P\left(M_{r}\right)$ over this scale range in the dissipation rate fields in Fig. 30 to be substantially different from that in the enstrophy fields in Fig. 29. The wider distribution of $M$ values in the enstrophy reflects a more intermittent structure in that field than for the dissipation field at the same $\operatorname{Re}_{\delta}$. This is consistent with the typical structures evident in both these fields in Figs. 22 and 26. A similar observation has been made from DNS investigations, ${ }^{39-41}$ and this is also consistent with previous estimates from one-dimensional measurements ${ }^{42}$ that the enstrophy field is more intermittent than the dissipation. It has been previously argued that on symmetry grounds the intermittency in both fields should be the same, ${ }^{43,44}$ and in any case that as $\operatorname{Re}_{\lambda} \rightarrow \infty$ the associated scaling exponents of both fields should become the same. The present direct measurements of both these fields show, however, that at least under these conditions in this turbulent shear flow the enstrophy field is clearly more intermittent than the dissipation rate field.

The scale similarity for $\left(r / \lambda_{v}\right) \geqslant 2.5$ in $P\left(M_{r}\right)$ in Figs. 29 and 30 relies on any remaining scale-to-scale variations being solely attributable to statistical convergence due to the finite number of independent one-dimensional intersections available from these measurements. This can be tested using the procedure in Ref. 45, where the scale-invariant form of $P(M)$ is used in a random multiplicative cascade to generate one-dimensional intersections having multifractal scale similarity. From these, corresponding $P\left(M_{r}\right)$ distributions are constructed, and the maximum allowable scale-to-scale difference norm $L_{1}\left(r / \lambda_{v}\right)$ between the distributions at successive $\left(r / \lambda_{v}\right)$ is obtained. These differences can then be compared with corresponding $L_{1}\left(r / \lambda_{\nu}\right)$ from the measured $P\left(M_{r}\right)$. Such results are shown in Fig. 31, which verify that the small scale-to-scale differences in the measured $P\left(M_{r}\right)$ distributions agree well with the limits due solely to incomplete statistical convergence, confirming that the dissipation field in turbulent flows displays multifractal scale similarity at scales $\left(r / \lambda_{\nu}\right) \geqslant 2.5$.

\section{DISCUSSION AND CONCLUSIONS}

The present results have experimentally documented numerous aspects associated with the structure, statistics, similarity and scaling of the turbulence at intermediate and small scales in turbulent shear flows. The mean strain rate values $\left(S \delta / u_{c}\right)=0$ and 1.7 investigated here correspond to $(S k / \varepsilon)$ 

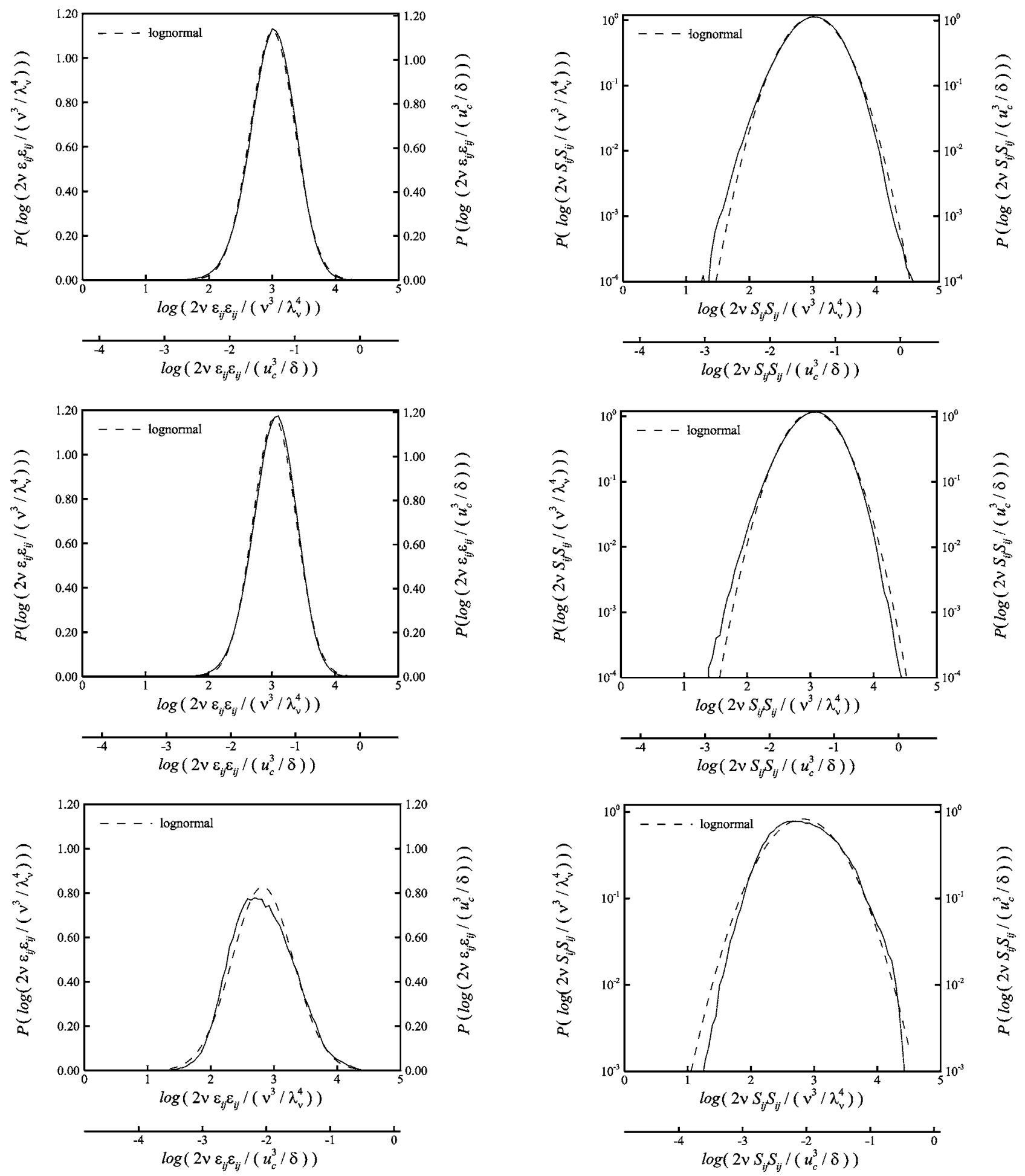

FIG. 27. Probability densities of the measured kinetic energy dissipation rates $2 \nu S_{i j} S_{i j}(\mathbf{x}, t)$, scaled on local inner $\left(\nu, \lambda_{\nu}\right)$ and outer $\left(u_{c}, \delta\right)$ variables, for $\left(\operatorname{Re}_{\delta} ; S\right)=(6,000 ; 0)$ (top), $(30,000 ; 0)$ (middle), and $(6,000,1.2)$ (bottom), with dashed lines giving log normal fits.

$=0$ and 2.1 , and represent the radial locations of maximum and minimum mean shear in an axisymmetric turbulent jet. This range of normalized mean shear rates is typical of that found in most other free turbulent shear flows, and throughout most wall-bounded turbulent shear flows as well, with values of $(S k / \varepsilon) \geq 3$ occurring only below the log layer in near-wall turbulence. The present results should thus be indicative of the approach to isotropy and the departures from isotropy at these scales in most turbulent shear flows.

Unlike uniformly sheared turbulence, in which the imposed mean shear rate is the same everywhere, in turbulent shear flows the mean shear rate varies substantially along the lateral direction, and the rapid lateral stirring created by the large-scale structures thus subjects the turbulence to a widely varying range of mean strain rates. Note that these large scales transport fluid laterally across the shear flow on a time 

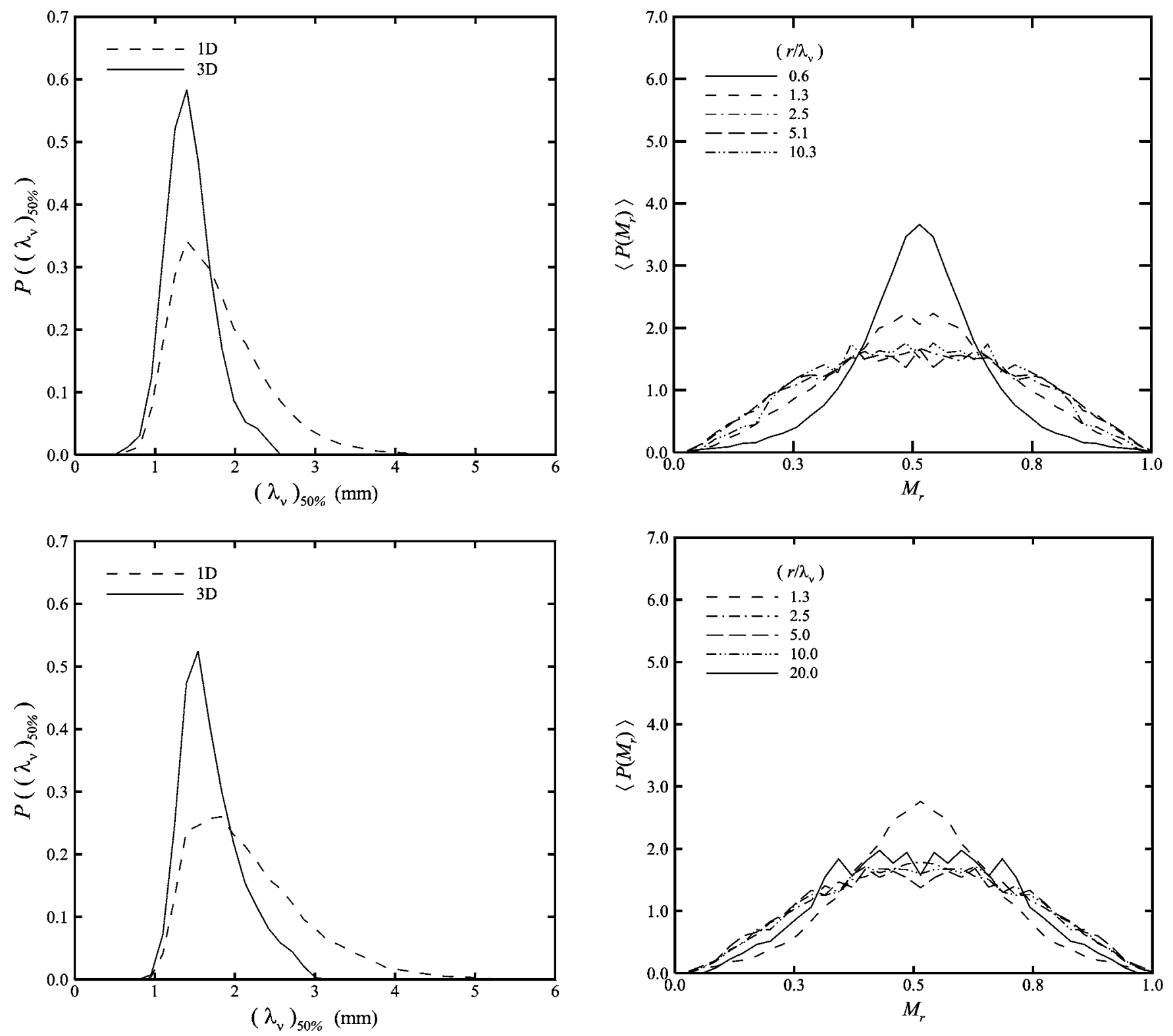

FIG. 28. Probability densities of inner-scale thicknesses $\left(\lambda_{\nu}\right)_{50 \%}$ obtained directly from one-dimensional intersections and from deconvolution of $\left(\lambda_{v}\right)_{1 \mathrm{D}}$ (dashed) to yield $\left(\lambda_{v}\right)_{3 \mathrm{D}}$ (solid) for the enstrophy field (top) and the kinetic energy dissipation rate field (bottom) at $\left(\operatorname{Re}_{\delta} ; S\right)=(6,000 ; 0)$.

scale $\left(\delta / u_{c}\right)$, which is seen in Table I to be about the same as the time scale $(k / \varepsilon)$ on which the turbulence can readjust to the changing mean strain rate. The turbulence in shear flows is thus only marginally able to maintain an equilibrium between the local mean strain field and the local turbulence properties. If the ratio $\Pi=\left(\delta / u_{c}\right) /(k / \varepsilon)$ of these time scales is in the limit $\Pi \gg 1$ then turbulence can maintain the equilibrium with the local mean strain field, and if $\Pi \ll 1$ the turbulence will be far out of equilibrium and essentially uncorrelated with the local strain field. A partial equilibrium exists for $\Pi \approx 1$, which applies throughout most turbulent shear flows. In this case the varying mean strain field to which the turbulence is subjected will principally affect the larger scales, which are in closer equilibrium with the local mean strain rate, while successively smaller scales are increasingly further out of equilibrium and thus should ap-
FIG. 29. Assessment of multifractal scale similarity from enstrophy field measurements at $\left(\operatorname{Re}_{\delta} ; S\right)=(6,000 ; 0)$ (top) and $(30,000 ; 0)$ (bottom), showing multiplier distributions $P\left(M_{r}\right)$ at various scales $\left(r / \lambda_{\nu}\right)$.

proach a state that is independent of the local mean strain rate.

The present results, which focus on the small scales, support such a partial equilibrium in turbulent free shear flows. They show clear differences in the level of anisotropy between the $\left(S \delta / u_{c}\right)=0$ and 1.7 cases, reflecting departures from the ideal equilibrium limit at these scales. The two $S$ $=0$ cases in Fig. 14, for instance, show significant anisotropy in the strain rate eigenvector orientations, in a way that reflects a residual signature of the much stronger anisotropy seen in the $S \neq 0$ case. This suggests that turbulence which had previously been at the radial location of maximum mean shear has, upon being transported to the centerline, undergone only a partial relaxation to the isotropic equilibrium state that would nominally apply at $S=0$ if the mean strain rate were not changing. Similar effects are seen in the vor- 

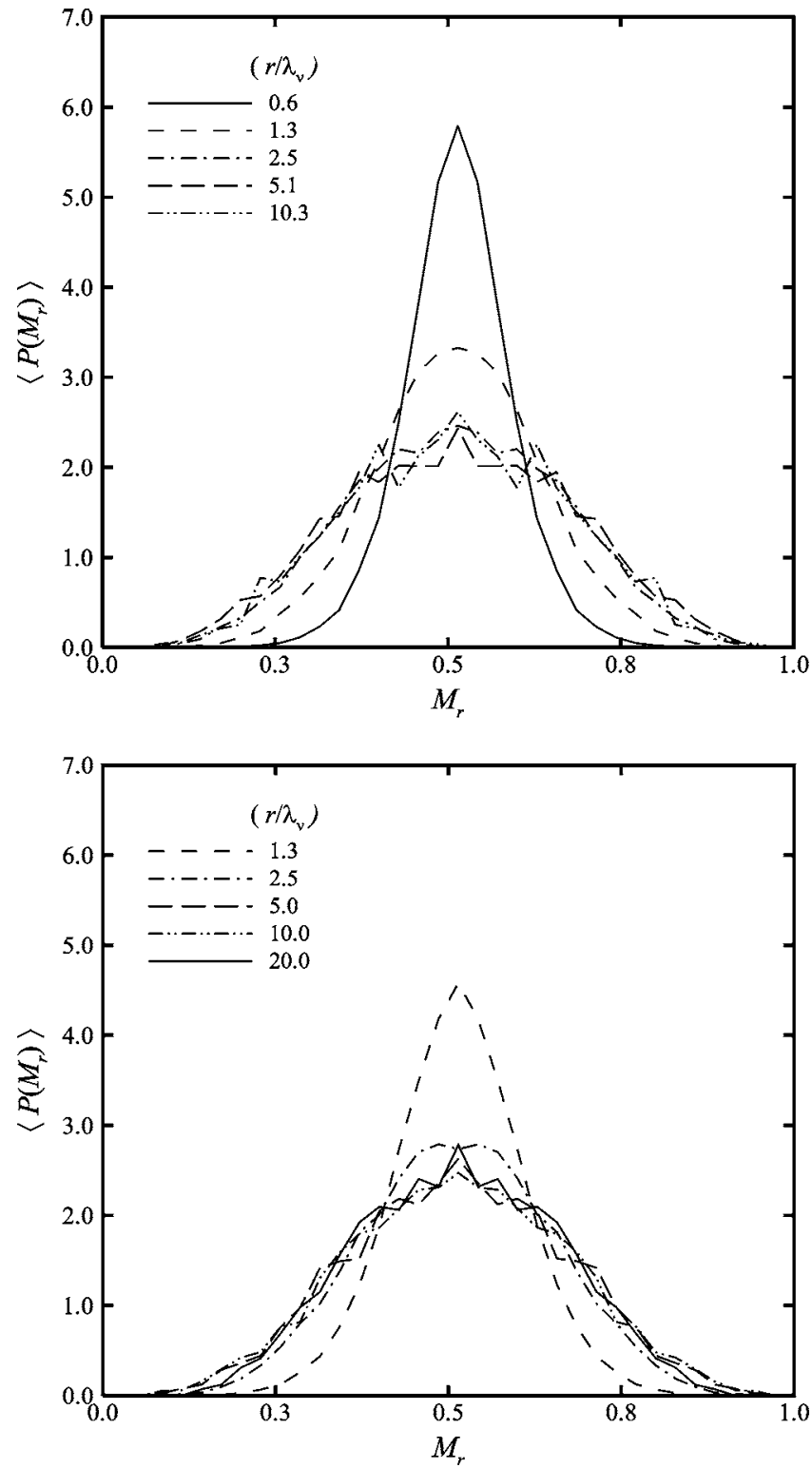

FIG. 30. Assessment of multifractal scale similarity from dissipation field measurements at $\left(\operatorname{Re}_{\delta} ; S\right)=(6,000 ; 0)$ (top) and $(30,000 ; 0)$ (bottom), showing multiplier distributions $P\left(M_{r}\right)$ at various scales $\left(r / \lambda_{\nu}\right)$.

ticity orientations in Figs. 19 and 21, and in the enstrophy production rates in Fig. 25.

Owing to the relative difficulty in making such highly resolved velocity gradient measurements at the small scales of turbulent shear flows, it is possible that some of the effects seen in these results may be due to small measurement errors. However, the extensive assessments in Ref. 18 of the accuracy in such measured velocity gradients suggest that these errors are sufficiently small. This is further supported by the strong similarity seen in the velocity gradients measured independently in this study at $\operatorname{Re}_{\delta}=6,000$ and 30,000 .

Key issues relevant to the partial equilibrium in shear flow turbulence include the effect of the local mean shear $\left(S \delta / u_{c}\right)$ on the range of scales over which significant departures from the small-scale equilibrium will exist, as well as the effect of the relative mean shear on the rate of approach to this equilibrium with decreasing scale size. The present

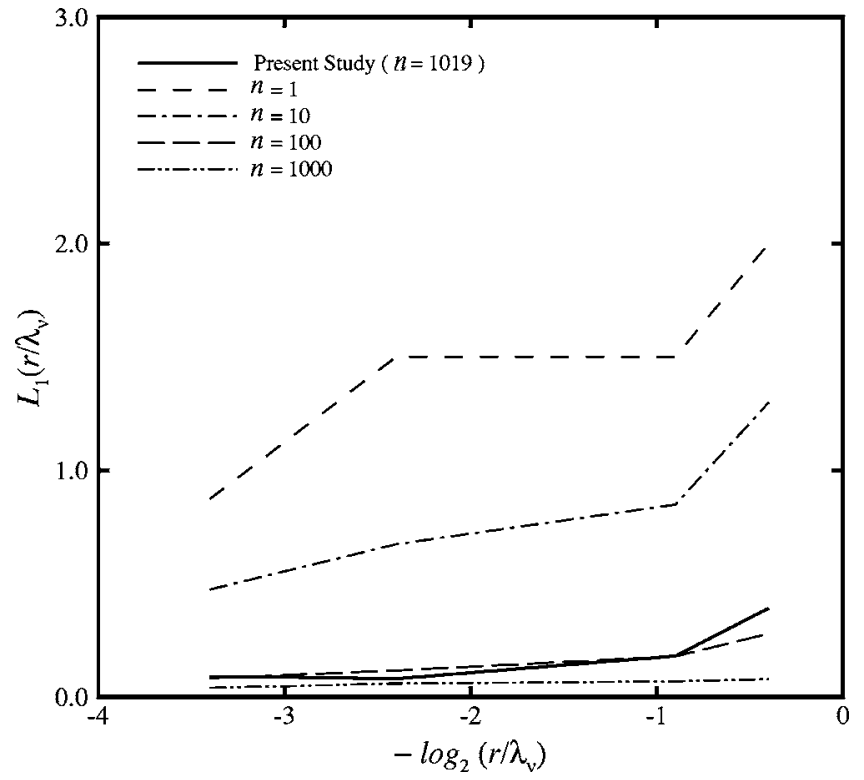

FIG. 31. Allowable scale-to-scale difference norms $L_{1}\left(r / \lambda_{\nu}\right)$ due to statistical convergence for various numbers $n$ of independent one-dimensional intersections through the dissipation field.

results show for $S \neq 0$ significant departures from the equilibrium state remain present even at the smallest scales for the Reynolds numbers currently accessible with these measurements. These $\operatorname{Re}_{\lambda}$ values, ranging from 45 to 113, are typical of early DNS investigations of homogeneous isotropic turbulence, and will likely increase as experimental measurement capabilities progress further. While DNS of homogeneous isotropic or uniformly sheared turbulence in periodic domains can reach far higher $\mathrm{Re}_{\lambda}$, comparable simulations of spatially developing turbulent shear flows are restricted to lower $\operatorname{Re}_{\lambda}$ by the need to simulate the entire range of flow scales, from the organized large-scale structures to the finest diffusive scales, over a sufficiently large spatial domain for the results to be unaffected by details of the inflow, outflow and boundary conditions. In the meantime, advanced measurement capabilities such as the dual-plane stereo particle image velocimetry method used in this study and in Refs. 46-52, as well as other single-point probe-based and multidimensional imaging-based measurement capabilities noted in Ref. 18, may provide the most direct means for probing these issues in turbulent shear flows.

\section{ACKNOWLEDGMENTS}

This work was jointly supported, in part, by the National Aeronautics and Space Administration (NASA) Marshall and Glenn Research Centers and the Department of Defense (DoD) within the NASA Constellation University Institutes Project (CUIP) Space Vehicle Technology Institute (SVTI) under Grant No. NCC3-989, with Claudia Meyer as the project manager. Discussions with Professor Nao Ninomiya are gratefully acknowledged, as is the assistance of Zac Nagel in conducting the measurements and Dr. Uwe Dierksheide and Dr. Callum Gray of LaVision, GmbH with the 
DSPIV acquisition software development. Dr. Cam Carter and Dr. Jeff Donbar of AFRL kindly provided the dye lasers.

${ }^{1}$ A. Pumir and B. I. Schraiman, "Persistent small scale anisotropy in homogeneous shear flows," Phys. Rev. Lett. 75, 3114 (1995).

${ }^{2}$ K. R. Sreenivasan and R. A. Antonia, "The phenomenology of small-scale turbulence," Annu. Rev. Fluid Mech. 29, 435 (1997).

${ }^{3}$ S. Garg and Z. Warhaft, "On the small scale structure of simple shear flow," Phys. Fluids 10, 662 (1998).

${ }^{4} \mathrm{~L}$. Biferale and M. Vergassola,"Isotropy vs anisotropy in small-scale turbulence," Phys. Fluids 13, 2139 (2001).

${ }^{5}$ K. A. Buch and W. J. A. Dahm, "Experimental study of the fine-scale structure of conserved scalar mixing in turbulent flows. Part 1 . Sc $\ll 1$," J. Fluid Mech. 317, 21 (1996)

${ }^{6}$ K. A. Buch and W. J. A. Dahm, "Experimental study of the fine-scale structure of conserved scalar mixing in turbulent flows. Part 2. Sc $\approx 1$, J. Fluid Mech. 364, 1 (1998).

${ }^{7}$ W. J. A. Dahm and K. B. Southerland, "Quantitative flow visualization via fully resolved four-dimensional spatio-temporal imaging," in Flow Visualization: Techniques and Examples, edited by A. Smits and T. T. Lim (Imperial College, London, 1999), pp. 231-258.

${ }^{8}$ L. K. Su and N. T. Clemens, "Planar measurements of the full threedimensional scalar dissipation rate in gas-phase turbulent flows," Exp. Fluids 27, 507 (1999).

${ }^{9}$ L. K. Su and N. T. Clemens, "The structure of fine-scale scalar mixing in gas-phase planar turbulent jets," J. Fluid Mech. 488, 1 (2003).

${ }^{10}$ C. A. Friehe, C. W. Van Atta, and C. H. Gibson, "Jet turbulence: dissipation rate measurements and correlations," AGARD Conf. Proc. CP-93, 18-1 (1971).

${ }^{11}$ M. Nelkin, "Universality and scaling in fully developed turbulence," Adv. Geophys. 43, 143 (1994).

${ }^{12}$ G. Stolovitsky, P. Kailasanath, P., and K. R. Sreenivasan, "Refined similarity hypotheses for passive scalars mixed by turbulence," J. Fluid Mech. 297, 275 (1995)

${ }^{13}$ W. J. A. Dahm, and K. B. Southerland, "Experimental assessment of Taylor's hypothesis and its applicability to dissipation estimates in turbulent flows," Phys. Fluids 9, 2101 (1997).

${ }^{14} \mathrm{M}$. M. Rogers and P. Moin, "The structure of the vorticity field in homogeneous turbulent flows," J. Fluid Mech. 176, 33 (1987).

${ }^{15} \mathrm{~S}$. Tavoularis and S. Corrsin, "Experiments in nearly homogeneous turbulent shear flow with a uniform mean temperature gradient. Part 1," J. Fluid Mech. 104, 311 (1981).

${ }^{16} \mathrm{M}$. Ferchichi and S. Tavoularis, "Reynolds number effects on the fine structure of uniformly sheared turbulence," Phys. Fluids 12, 2942 (2000).

${ }^{17}$ J. A. Mullin and W. J. A. Dahm, "Dual-plane stereo particle image velocimetry (DSPIV) for measuring velocity gradients at intermediate and small scales of turbulent flows," Exp. Fluids 38, 185 (2005).

${ }^{18}$ J. A. Mullin and W. J. A. Dahm, "Dual-plane stereo particle image velocimetry measurements of velocity gradient tensor fields in turbulent shear flow. I. Accuracy assessments," Phys. Fluids 18, 035101 (2006).

${ }^{19} \mathrm{~S}$. Biringen, "An experimental investigation of an axisymmetric jet issuing into a coflowing stream," VKI Technical Note 110 (1975).

${ }^{20}$ T. B. Nickels and A. E. Perry, "An experimental and theoretical study of the turbulent coflowing jet," J. Fluid Mech. 309, 157 (1996).

${ }^{21}$ J. A. Mullin, "A study of velocity gradient fields at the intermediate and small scales of turbulent shear flows via dual-plane stereo particle image velocimetry," Ph.D. dissertation, The University of Michigan, Ann Arbor, MI (2004)

${ }^{22}$ J. Jimenez, A. A. Wray, P. G. Saffman, and R. S. Rogallo, "The structure of intense vorticity in isotropic turbulence," J. Fluid Mech. 255, 65 (1993).

${ }^{23}$ T. Gotoh, D. Fukayama, and T. Nakano, "Velocity field statistics in homogeneous steady turbulence obtained using a high-resolution direct numerical simulation," Phys. Fluids 14, 1065 (2002).

${ }^{24}$ A. Tsinober, E. Kit, and T. Dracos, "Experimental investigation of the field of velocity gradients in turbulent flows," J. Fluid Mech. 242, 169 (1992).

${ }^{25} \mathrm{~T}$. Gotoh, "Probability density functions in steady-state Burgers turbulence," Phys. Fluids 11, 2143 (1999).

${ }^{26}$ B. Tao, J. Katz, and C. Meneveau, "Statistical geometry of subgridscale stresses determined from holographic particle image velocimetry measurements," J. Fluid Mech. 457, 35 (2002).

${ }^{27}$ T. S. Lund and M. M. Rogers, "An improved measure of strain rate probability in turbulent flows," Phys. Fluids 6, 1838 (1994).
${ }^{28}$ W. T. Ashurst, A. R. Kerstein, R. M. Kerr, and C. H. Gibson, "Alignment of vorticity and scalar gradient in simulated Navier-Stokes turbulence," Phys. Fluids 30, 2343 (1987).

${ }^{29}$ L. K. Su and W. J. A. Dahm, "Scalar imaging velocimetry measurements of the velocity gradien tensor field in turbulent flows. I. Assessment of errors," Phys. Fluids 8, 1869 (1996).

${ }^{30}$ L. K. Su and W. J. A. Dahm, "Scalar imaging velocimetry measurements of the velocity gradient tensor field in turbulent flows. II. Experimental results," Phys. Fluids 8, 1883 (1996).

${ }^{31}$ L. Ong and J. M. Wallace, "Joint probability density analysis of the structure and dynamics of the vorticity field of a turbulent boundary layer," J. Fluid Mech. 367, 291 (1998).

${ }^{32}$ J. Jimenez, "Kinematic alignment effects in turbulent flows," Phys. Fluids A 4, 652 (1992)

${ }^{33} \mathrm{~V}$. Yakhot, "Pressure-velocity correlations and scaling exponents in turbulence," J. Fluid Mech. 495, 135 (2003).

${ }^{34}$ W. J. A. Dahm and K. A. Buch, "Lognormality of the scalar dissipation pdf in turbulent flows," Phys. Fluids A 1, 1290 (1989).

${ }^{35} \mathrm{~K}$. A. Buch, "Fine scale structure of conserved scalar mixing in turbulent shear flows: $\mathrm{Sc} \gg 1, \mathrm{Sc} \approx 1$ and implications for reacting flows," Report No. 026779-5, Department of Aerospace Engineering, The University of Michigan (1991) .

${ }^{36}$ C. Meneveau and K. R. Sreenivasan, "Simple multifractal cascade model for fully developed turbulence," Phys. Rev. Lett. 59, 1424 (1987).

${ }^{37}$ C. Meneveau and K. R. Sreenivasan, "The multifractal nature of the turbulent energy dissipation,” J. Fluid Mech. 224, 429 (1991).

${ }^{38}$ K. R. Sreenivasan, "Fractals and multifractals in fluid dynamics," Annu. Rev. Fluid Mech. 23, 539 (1991).

${ }^{39}$ E. Siggia, "Numerical study of small-scale intermittency in threedimensional turbulence," J. Fluid Mech. 107, 375 (1981).

${ }^{40} \mathrm{R}$. Kerr, "Higher-order derivative correlations and the alignment of smallscale structures in isotropic numerical turbulence," J. Fluid Mech. 153, 31 (1985).

${ }^{41}$ S. Chen, K. R. Sreenivasan, and M. Nelkin, "Inertial range scalings of dissipation and enstrophy in isotropic turbulence," Phys. Rev. Lett. 79, 1253 (1997)

${ }^{42}$ C. Meneveau, K. R. Sreenivasan, P. Kailasnath, and M. S. Fan, "Joint multifractal measures: Theory and applications to turbulence," Phys. Rev. A 41, 894 (1990).

${ }^{43}$ V. L'vov and I. Procaccia, "The universal scaling exponents of anisotropy in turbulence and their measurement," Phys. Fluids 8, 2565 (1996).

${ }^{44}$ M. Nelkin, "Enstrophy and dissipation must have the same scaling exponent in the high Reynolds number limit of fluid turbulence," Phys. Fluids 11, 2202 (1999)

${ }^{45}$ R. D. Frederiksen, W. J. A. Dahm, and D. R. Dowling, "Experimental assessment of fractal scale similarity in turbulent flows. Part 3. Multifractal scaling," J. Fluid Mech. 378, 127 (1997).

${ }^{46}$ C. J. Kähler and J. Kompenhans, "Multiple plane stereo PIV: Technical realization and fluid mechanical significance," in Proceedings of the 3rd International Workshop on PIV, Santa Barbara (1999).

${ }^{47}$ H. Hu, T. Saga, T. Kobayashi, N. Taniguchi, and M. Yasuki, "Dual-plane stereoscopic particle image velocimetry: System set-up and its application on a lobed jet mixing flow," Exp. Fluids 31, 277 (2001).

${ }^{48}$ J. A. Mullin and W. J. A. Dahm, "Highly resolved three-dimensional velocity measurments via dual-plane stereo particle image velocimetry (DSPIV) in turbulent flows," AIAA Paper No. 2002-0290 (2002).

${ }^{49}$ C. J. Kähler, M. Stanislas, and J. Kompenhans, "Spatio-temporal flow structure investigation of near-wall turbulence by means of multiplane stereo particle image velocimetry," in Proceedings of the 11th International. Symposium on Applications of Laser Technology to Fluid Mechanics, Lisbon, Portugal (2002).

${ }^{50}$ J. A. Mullin and W. J. A. Dahm, "Dual-plane stereo PIV (DSPIV) measurements of the velocity gradient tensor field at the small scales of turbulent flows," in Proceedings of the 3rd International Conference on Turbulence and Shear Flow Processes, Sendai, Japan (2003).

${ }^{51}$ C. J. Kähler, "Investigation of the spatio-temporal flow structure in the buffer region of a turbulent boundary layer by means of multiplane stereo PIV," Exp. Fluids 36, 114 (2004).

${ }^{52}$ B. Ganapathisubramani, E. K. Longmire, I. Marusic, and S. Pothos, "Dual-plane PIV technique to resolve complete velocity gradient tensor in a turbulent boundary layer," in Proceedings of the 12th International Symposium on Applied Laser Technology to Fluid Mechanics, Lisbon, Portugal (2004). 